\title{
Building Roof Superstructures Classification from Imbalanced and Low Density Airborne LiDAR Point Cloud
}

\author{
Baha Eddine Aissou, Aichouche Belhadj Aissa, Abdelkader Dairi, Fouzi Harrou, Andreas Wichmann, Martin \\ Kada
}

\begin{abstract}
Light Detection and Ranging (LiDAR), an active remote sensing technology, is becoming an essential tool for geoinformation extraction and urban planning. Airborne Laser Scanning (ALS) point clouds segmentation and accurate classification are challenging and crucial to produce different geoinformation products like three-dimensional (3D) city designs. This paper introduces an effective data-driven approach to build roof superstructures classification for airborne LiDAR point clouds with very low density and imbalanced classes, covering an urban area. Notably, it focuses on building roof superstructures (especially dormers and chimneys) and mitigating nonplanar objects' problems. Also, the imbalanced class problem of LiDAR data, to the best of our knowledge, is not yet addressed in the literature; it is considered in this study. The major advantage of the proposed approach is using only raw data without assumptions on the distribution underlying data. The main methodological novelties of this work are summarized in the following key elements. (i) At first, an adapted connected component analysis for 3D points cloud is proposed. (ii) Twelve geometry-based features are extracted for each component. (iii) A Support Vector Machine (SVM)-driven procedure is applied to classify the 3D components. (iv) Furthermore, a new component size-based sampling (CSBS) method is proposed to treat the imbalanced data problem and has been compared with several existing resampling strategies. In this study, components are classified into five classes: shed and gable dormers, chimneys, ground, and others. The results of this investigation show the satisfying classification performance of the proposed approach. Results also showed that the proposed approach outperformed machine learning methods, including SVM, Random Forest, Decision Tree, and Adaboost.
\end{abstract}

Index Terms-Low-density point cloud, light detection and ranging (LiDAR), roof superstructures, 3D classification, imbalanced data.

Manuscript received March 25, 2021. This work was supported by the Technische Universität Berlin.

B. Aissou and A. Belhadj Aissa are with Image Processing and Radiation Laboratory, University of Sciences and Technology Houari Boumediene, USTHB, Bp 32 El Alia, Bab Ezzouar Alger, 16111, Algerie. (E-mail: bahae.aissou@gmail.com, houria.belhadjaissa@gmail.com).

A. Dairi is with Computer Science Department, University of Oran 1 Ahmed Ben Bella , Algeria Street El senia el mnouer bp 31000 Oran, Algeria. (E-mail: dairi.aek@gmail.com).

F. Harrou is with King Abdullah University of Science and Technology (KAUST) Computer, Electrical and Mathematical Sciences and Engineering (CEMSE) Division, Thuwal, 23955-6900, Saudi Arabia, E-mail: fouzi.harrou@kaust.edu.sa

A. Wichmann is with LGLN Landesamt für Geoinformation und Landesvermessung Niedersachsen, Podbielskistraße 331, 30659 Hanover, Germany. (E-mail: andreas.wichmann@lgln.niedersachsen.de).

M. kada is with Institute of Geodesy and Geoinformation Science, Technische Universität Berlin, Straße des 17. Juni 135, 10623 Berlin, Germany. (E-mail: martin.kada@tu-berlin.de).

\section{INTRODUCTION}

Airborne Light Detection and Ranging (LiDAR), also called Airborne Laser Scanning (ALS), is an active sensor used in remote sensing techniques for extracting various information about physical surfaces. ALS provides dense, discrete, detailed, and accurate three-dimensional (3D) point clouds that directly capture objects and ground surfaces [1].

Over the past decade, LiDAR point clouds have become an essential tool in several applications, including 3D Reconstruction of building model [2], [3], Forest management [4], [5], Navigation and localization in dynamic environments [6], [7], place recognition [8], instruments modeling [9], [10], creation of digital terrain models (DTM) [11] and topographic mapping for digital city [12]. The raw measurements of point cloud data can include one or several echoes, depending on the sensor technology. Note that other products can be derived from point clouds, such as generating digital elevation models (DEM) [13] and digital surface models (DSM) [14]. The derived product exploitation enables using well-known processing image methods; however, the transition from 3D point cloud to $2.5 \mathrm{D}$ results in a loss of information. Essentially, using the raw point cloud involves more sophisticated techniques and the advantage of preserving 3D spatial information.

Designing an efficient methodology for urban area classification based on the raw LiDAR point cloud data is very important from the practical point of view [15]. Towards this end, there are two classes of methods: point-based and segment-based classification [16]. The essence of pointbased classification is to compute features for each point; thus, the class label is assigned point by point. On the other hand, segment features are computed by averaging the feature values of the points in a segment, making it more discriminative compared to the point-based one [17].

Generally speaking, 3D point cloud segmentation methods can be categorized into planar and nonplanar methods. Surface segmentation from point cloud is usually performed by surface growing [18], 3D Hough transform [19], or random sample consensus (RANSAC) [20]. Recently, the same methods are used to extract planar surfaces, such as roof faces and other surfaces from ALS data [21], [22]. However, they are not suited to segment nonplanar objects with free form shape. For nonplanar surfaces, Melzer [23] applied a 
mean shift algorithm for urban point cloud segmentation into buildings, vegetation, and terrain [24]. Rutzinger et al. [25] considered segment growing based on echo width homogeneity for clustering neighboring vegetation points in an urban environment from full-waveform ALS data. It has been shown that the application of one single segmentation method for LiDAR data typically does not provide satisfactory results, especially in urban areas [17]. Thus, it is interesting to combine planar and nonplanar segmentation methods to identify a maximum of entities. For this purpose, large surface segments, such as buildings roofs, are often extracted with a planar segmentation method, and the remaining points with a nonplanar process.

The segmentation of nonplanar objects, such as roof superstructures, remains an ongoing issue for ALS data because it causes the division of a large building roof part into several small segments [26]. Specifically, gaps appear in the building roof point cloud when the laser beam does not return a pulse due to openings in a roof (i.e., holes due to occluded building roof parts). Vegetation close to the roof or roof superstructures, such as dormers and chimneys, can create these openings that appear as penetrable surfaces [27]. Indeed, the great variety of geometrical shapes of dormers and chimneys makes their identification a challenging task. This difficulty can increase further with lowdensity point clouds. It is recommended to handle highdensity point clouds in urban areas because of the large number of details. The benefit of decreasing point cloud density is reducing data acquisition costs and bypassing calculation problems usually encountered with high-density data. For 3D building details, usually, a density higher than ten points $/ \mathrm{m}^{2}$ is needed. The use of a lower density point cloud necessitates a powerful clustering technique and relevant features to discriminate building roof details. Accordingly, researchers tend to omit dormers and chimneys even if ubiquitous in an urban area, leading to poor roof extraction. In [28], Kada and Wichmann introduced subsurface growing (SSG) to deal with the problem of holes caused by roof superstructures. To this end, they merge nearby segments to avoid the division of large segments into smaller patches. This allows the general roof structure to be reconstructed whereas excluding roof superstructures details (e.g., dormers and chimneys). In [29], Zolanvari et al. proposed an Improved Slicing Method (ISM) to detect openings in the roofs even in the presence of chimneys. However, the ISM approach does not consider dormers which are more numerous on the roofs than chimneys. In [30], Fan et al. considered the points of chimneys as noise because they are small-sized. Note that the small size of dormers and chimneys significantly affects their detection. In [9], Xu et al. concluded that small segments often lead to misclassification because of inaccurate features. In [31], Maas and Vosselman explicitly reconstruct dormers from original ALS data. However, small dormer reconstruction posed problems due to unrealistic assumptions about the number of points on the dormers and their orientation. In [32], Brédif et al. described an automatic method for detecting 3D building models, including dormers, by using a collection of parametric models of the available dormer types. However, the limitation of this method is the complexity of finding the best fitting model among the collection.

This paper aims to highlight the roof superstructures, often ignored or considered unimportant objects in the literature. To this end, holes in building roofs are restored to provide a realistic final output. Moreover, this study alleviates the issue of imbalance classes in LiDAR data classification. Note that supervised learning classifiers require roughly balanced classes in the learning stage; that is, each class has sufficient representative instances in the training set [33]. Thus, any dominance of a class will affect the training stage and results in misleading classification results; the majority of instances will take the dominant class label [34]. More specifically, most research focuses on the dominant classes of interest and tends to discard the small classes. In a complex urban area, targets are neither evenly distributed nor cover similar area proportions of the studied area. It is unavoidable to record a dissimilar fraction of instances from each class when using an ALS [35].

The main contributions of this study are summarized in the following key elements.

- Five classes from LiDAR point clouds are considered in this study: two dormer types (shed and gable), chimneys, ground, and the class others, making it more challenging than most LiDAR classification studies. The existing methods practically ignore this detailed information and focus on large classes (ground, building, and vegetation).

- A low-density point cloud in an urban area, representing the points not assigned by subsurface growing, is exploited. It is worth pointing out that the proposed approach is flexible and does not require additional data or model assumptions, which can be considered extreme conditions. For this purpose, connected component analysis is proposed to cluster raw point cloud to avoid information losses. Moreover, twelve geometry-based features are introduced from the components to discriminate the target classes composed of both nonplanar (dormers and chimneys) and planar objects (ground). Then, a correlation-based feature selection procedure is applied to identify the eight relevant features.

- Furthermore, this study mitigates the problem of imbalanced instances and classes of interest with a low probability. Here, the component size-based sampling method is proposed and compared with different synthetic sampling strategies. To that extend, interactive labeling is performed to form the training data. The performance of the proposed approach is compared to conventional classifiers for LiDAR data, including SVM, Random Forest, Decision Tree, and Adaboost. Real ALS point cloud data set provided by the German Society for Photogrammetry, Remote Sensing and Geoinformation (DGPF) [36] is used to evaluate the 
proposed method's performance. The ALS data used is characterized by a low density $\left(4-6\right.$ points $\left./ \mathrm{m}^{2}\right)$. It covers an urban area located in residential parts of Vaihingen city in southern Germany. The classification accuracy has been assessed using six statistical indicators: overall accuracy, recall, precision, F1-score, and Area Under Curve (AUC). Despite the extremely considered conditions, the results show the relevance of the proposed method to overcome the problem of the holes due to roof superstructures abundant in urban areas. This study could constitute a promising way to address the problem of scarcity of labeled LiDAR data.

This paper consists of four sections. Section II provides an overview of the preliminary materials, and Section III introduces the proposed methodology. Section V assess the proposed method and compare its performance with traditional methods. Finally, Section VI concludes this study and sheds light on potential future research lines.

\section{Preliminary materials}

This section provides an overview of the SSG method, connected component analysis for point cloud, and resampling procedures to rebalance the classes.

\section{A. Subsurface Growing}

Two-dimensional (2D) segmentation methods are usually well suited to segment planar roof segments if only simple roofs without roof superstructures (e.g., dormers, chimneys, or ventilations) are present. Otherwise, the extracted roof segments increase in number while becoming smaller with a more complex shape. Consequently, the segmentation of the underlying roof surface becomes more complex (Fig. 1(a)). As indicated, common segmentation methods based on surface growing would usually return fivesegment patches for the main roof's front side. The major reason is that the segment patches' growth phase has been prematurely ended due to the dormers located between them. In [28], subsurface growing has been introduced as an extension of surface growing to overcome this issue. The basic idea of subsurface growing is to incorporate the measured surface points of an ALS data set during the segmentation process and create and consider virtual points located below the real roof surface. The extension consists of the following two steps: creating virtual points in (1), and merging of neighboring segments in (2). Specifically, the creation of virtual points is generally not limited in number, but they must be located under real point measurements and must be close to a plane represented by the measured points of a segment. Thereby, segments can grow below other segments to avoid the aforementioned division of large segments into smaller patches. Indeed, two neighboring segments $S_{1}$ and $S_{2}$ are merged to the segment $S_{\text {new }}$ if they meet the following two criteria:

$$
\begin{aligned}
& \nexists p_{1}, p_{2} \mid \operatorname{distance}\left(p_{1}, S_{\text {new }}\right)>\theta_{D} \\
& \wedge \operatorname{distance}\left(p_{2}, S_{\text {new }}\right)>\theta_{D}
\end{aligned}
$$

$$
\begin{aligned}
& \text { angle }\left(\text { normal }_{S_{1}}, \text { normal }_{S_{\text {new }}}\right)<\theta_{A} \\
& \left.\wedge \operatorname{angle} \text { normal }_{S_{2}}, \text { normal }_{S_{\text {new }}}\right)<\theta_{A}
\end{aligned}
$$

where $p_{1}$ and $p_{2}$ are the measured point of $S_{1}$, and $S_{2}$ respectively. $\theta_{D}$ and $\theta_{A}$ are chosen threshold values. Fig. 1(b) shows the result of the subsurface growing method for the previous example. Small roof details usually lost due to their low number of points are now a part of larger segments. Although virtual points can improve the segmentation result so that the resulting segments become larger and more stable, many measured points belonging to roof superstructures are usually not assigned to any segment during the surface growing phase.

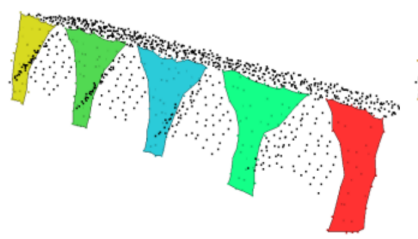

(a)

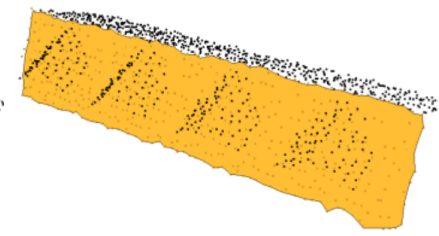

(b)
Fig. 1: Planar segments of the base roof front surface: (a) as a result of surface growth, and (b) by applying sub-surface growing.

\section{B. Connected Component Analysis}

Connected components analysis (or connected components labeling) is a clustering algorithm widely used in image processing. For instance, in [37], Miliaresis et al. applied connected component labeling to identify the background and foreground objects from DEMs. In [38], Rubio et al. exploited connected components from the original point cloud instead of derived products such as DEMs to group points. In [39], Zhang et al. employed connected components for point clouds to improve the classification of LIDAR data in urban areas. In [40], Xiao et al. considered a connected components algorithm for clustering tree points. However, points within a certain distance, e.g., $2 \mathrm{~m}$ in 3D, are connected into one component. In [41], Vosselman used k-nearest neighbors as a clustering algorithm for connected component analysis of point clouds. Using a neighborhood size of 20 points implies that all points in a connected component of 20 or fewer will have the same feature values.

\section{Resampling strategy}

Imbalanced instances in the LiDAR data set are unavoidable, which significantly affects the classifier learning process. There are different approaches to overcome imbalanced data, including sampling strategies and synthetic data generation. Sampling methods include random undersampling and random oversampling techniques.

1) Random Undersampling: It consists of reducing the size of the majority (i.e., dominant) classes by randomly removing instances from the overrepresented classes until their size is equal to the smallest class. 
2) Random Oversampling: The essence of this resampling procedure is to increase the number of underrepresented classes by randomly duplicating their instances until their size is equal to the dominant class.

The synthetic data generation approach aims to handle the imbalance in the original data sets not by duplicating instances but by artificially generating data samples [42]. For this purpose, various techniques were proposed.

3) SMOTE approach: Chawla et al. introduced the Synthetic Minority Oversampling Technique (SMOTE) method [43]. In this approach, the minority class is oversampled by introducing synthetic examples representing $k$ nearest neighbors of each minority class instance.

4) Borderline-SMOTE approach: This approach is introduced as an extension of the SMOTE, where only the minority examples near the borderline samples are detected and used to generate new synthetic samples [44]. This is because the borderline examples of the minority class are easily misclassified compared to those far from the borderline.

5) ADASYN approach: The adaptive Synthetic Sampling Approach (ADASYN) aims to reduce the learning bias by generating synthetic data samples. ADASYN uses a weighted distribution for different minority class examples according to their level of difficulty in learning, where more synthetic data is generated for minority class examples that are harder to learn compared to majority examples [42].

6) SVMSMOTE approach: The SVMSMOTE is proposed in [45] based on oversampling borderlines minority class examples. In SVMSMOTE, a Support Vector Machine (SVM) classifier is then trained to predict future instances.

7) KMEANSSMOTE approach: Douzas and al. [46] developed the KMEANSSMOTE procedure as another variant of SMOTE method. Essentially, it consists of clustering the instances with the well-known Kmeans clustering algorithm before applying oversampling. This enables reducing the noisy samples and overcomes imbalances between classes. However, the tests were conducted based on an artificially simulated data set.

8) SMOTETOMEK: In [47], the SMOTETOMEK approach was introduced by combining oversampling and undersampling strategies. Oversampling is performed using SMOTE technique, while Tomek Link is used as an undersampling method for cleaning overlapping samples.

\section{Methodology}

Fig. 2 illustrates the flowchart of the proposed building roof superstructures classification procedure. The input data consists of raw LiDAR points unassigned by the subsurface growing segmentation method. The main steps performed in this methodology are summarized as follows. (1) A clustering algorithm using connected components analysis is conducted. (2) Twelve geometric-based features are computed for each component. (3) According to the corresponding class, interactive labeling is performed by draping the resulting components on an orthophoto and labeling them one by one. (4) A segment-based classification approach is applied where an SVM classifier with Radial Basic Function (RBF) kernel is used. (5) A new component size-based sampling method (CSBS) is proposed to deal with the problem of the imbalanced classes, and it is compared with synthetic sampling methods of literature. (6) Finally, to show the potential of the proposed method, the most used classifiers for LiDAR data (SVM, Random Forest, Decision tree, and Adaboost) are tested and compared using the best eight selected features.

\section{A. 3D Connected Component Analysis}

Here, we present an extension of the work given in [48]. In short, the principal characteristic of the proposed method consists of its flexibility in selecting neighbors. Specifically, when selecting neighbors, the algorithm does not restrict to the immediate neighborhood of the points but extends calculations until no points are found. In other words, it is different from that proposed in [49], where only the immediate neighbors are considered as a connected component. For each point belonging to the set, a label is assigned with the current label symbol, and then the point is removed from the set. One can imagine a 3D sphere with a radius equal to a predefined distance and the center's current point. If there are other points within the sphere, then these points are added to the component, and their label is updated with the same label as the current point. The process continues with the newly added points until no further neighboring points can be found. Thus, it will be sure that the entire domain will be treated. A similar method is presented in [39] which used a k-d tree and a fixed distance neighborhood to search a local neighborhood for each point of point clouds. However, constructing a k-d tree can lead to a too slow treatment for dense point clouds. Fig. 3 illustrates an example of a 3D connected component analysis result using Euclidean distance of $2 \mathrm{~m}$, considering only components with a size greater than four points. The resulting components (see Fig. 3(b)) are differently colored to illustrate the various objects. By considering the L2 norm, the steps of the proposed connected components are presented in Algorithm 1.

\section{B. Features Computing}

The low density of the input point cloud and the particularity of the target classes (i.e., dormers, chimneys) require the extraction of relevant features. For this purpose, twelve geometric-based features are proposed:

Feature 1: The size of the component represents the number of points belonging to it. The segment size was used to separate between vegetation and roof faces in [16], [17]. It can be helpful to distinguish between the classes roof superstructures and "others".

Feature 2: The component's maximum height difference represents the difference between the highest and lowest point in the component [50]. It should have a significant 


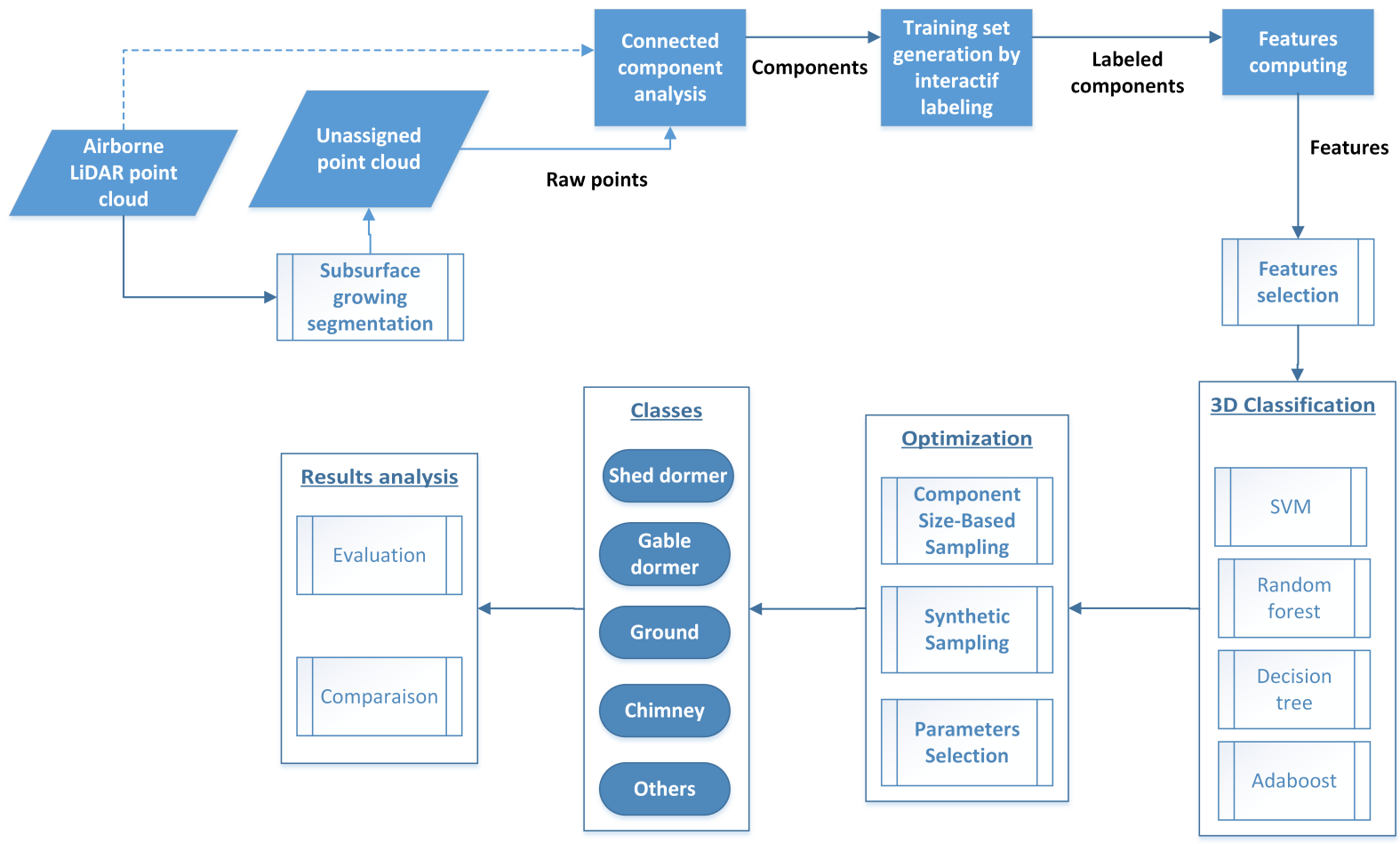

Fig. 2: The flowchart of the proposed classification strategy.

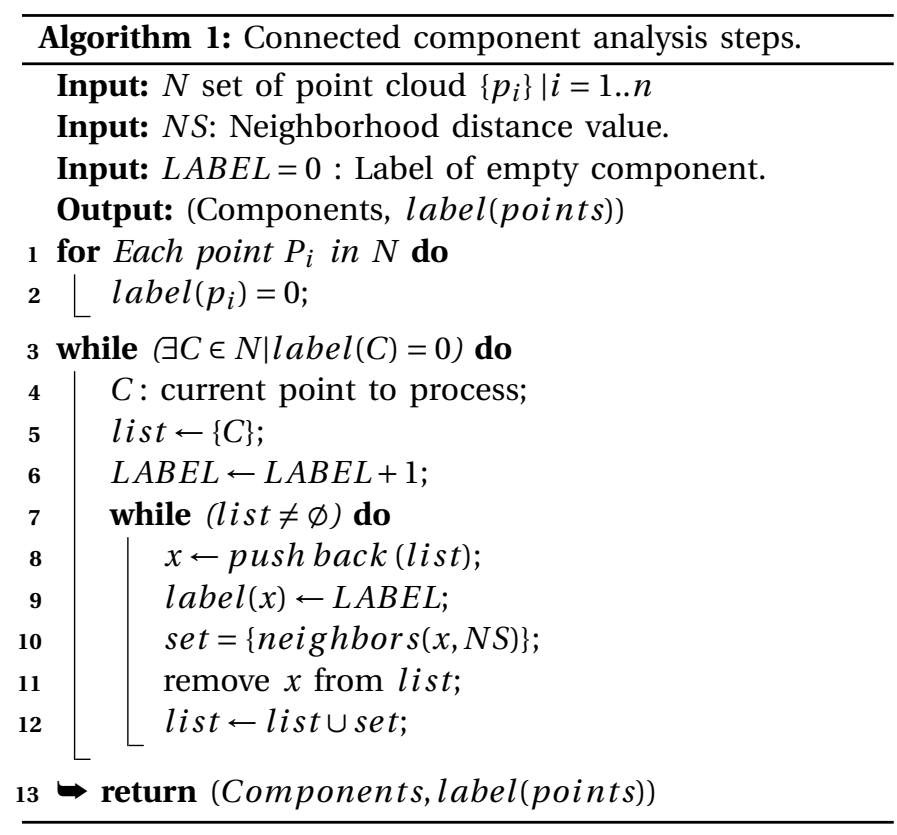

value for chimney components compared to a ground component, where the feature value typically should tend to zero unless it is on a steep slope.

Feature 3: The difference between the highest point in building, where belong the component, and the highest point in the component. This feature is introduced to discriminate the classes ground and chimney. When using only feature 2 , a component with similar height points can be classified as ground even if it is located on the roof. Feature 3 will have a great value in the case of the ground class and negative values in chimneys because those are located on the rooftop.

Feature 4: Each type of dormer and chimney has its shape that geometric features can characterize. These different shapes inspired us to compute the area of each region by constructing a 2D convex hull and 2D alpha shape (feature 5). The advantage of using the convex hull method is to find the geometrical area of each object with minimum computational effort [51].

Feature 5: The 2D alpha shape area is computed for each component to obtain discriminative and invariant features. The alpha shape is a linear approximation of the original shape from an unorganized set of points [52]. The definition of alpha shapes is based on an underlying triangulation. Circles with a fixed radius $(\alpha)$ approach the data points until they touch the points on the triangle edges [40].

Feature 6, Feature 7 and Feature 8: Three other features are used based on elevation, representing respectively the lowest and highest point in the component and the height average component points [15], [53]. Two additional features are introduced to express entropy information. Claude Shannon defined entropy information to represent the amount of information or uncertainty delivered by a random variable. Given a random variable $X$ with possible values $x_{i}$, each with probability $P_{X}\left(x_{i}\right)$. The entropy $H(x)$ of $\mathrm{x}$ is defined in (3).

$$
H(X)=-\sum_{i} P_{X}\left(x_{i}\right) \log _{b} P_{X}\left(x_{i}\right)=\sum_{i} P_{X}\left(x_{i}\right) I_{X}\left(x_{i}\right)
$$

where $I_{X}$ is the self-information of the random variable $X, I_{X}\left(x_{i}\right)$ is the self-information associated with a particular value, and $b$ the base of the logarithm. Entropy was 


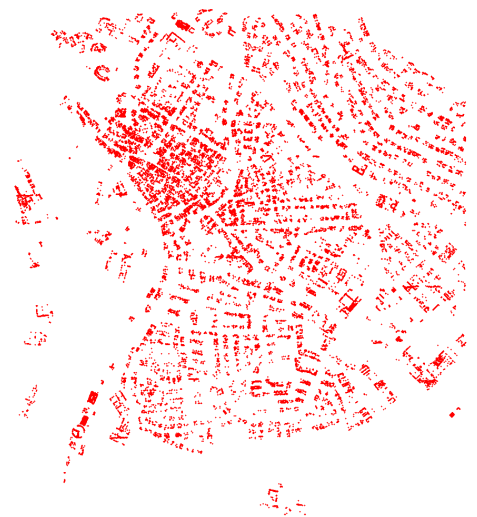

(a)

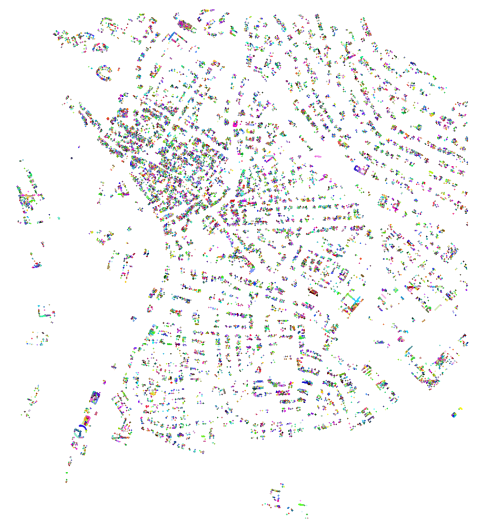

(b)

Fig. 3: Application of connected component analysis: (a) Raw points and (b) Connected components considering neighboring distance of $2 \mathrm{~m}$.

widely used for images derived from point cloud [36], [50]; however, to the best of our knowledge, no work considers entropy from raw point cloud measurements.

Feature 9: The Self-information of component size represents the information brought by the component's size in relation to the size of the building to which the component belongs.

Feature 10: represents the elevation entropy in the component and serves to quantify the degree of uncertainty provided by the heights of points constituting the component [54]. It is defined by:

$$
H(X)=-\sum_{i=1}^{N}\left(\frac{Z_{p i}}{\sum_{j=1}^{N} Z_{p j}} \log _{2} \frac{Z_{p i}}{\sum_{j=1}^{N} Z_{p j}}\right)
$$

where $N$ is the number of points in the component (i.e., size of the component), $p i$ and $p j$ are points belonging to the set of points forming the component.

Feature 11 and Feature 12: They represent standard deviation and coefficient variation of height in [15], introduced to characterize the dispersion of points in components. Table I summarizes the features introduced in this study.

\section{Component Size-Based Sampling Method}

The proposed CSBS method for rebalancing the classes is a hybrid method, which involves oversampling and undersampling techniques. The samples (i.e., instances) represent the component features. A component is a set of points clustered by connected component analysis. Essentially, features are the descriptors computed to discriminate the components. The component size represents the number of points included in the given component. The essence of the CSBS approach is that component points share similar features characterizing the component they belonging to it. This enables to oversample each sample considering its component size. Consequently, each sample grows according to the component size it represents. This is different from random oversampling, which considers the biggest class to oversample the classes. Thus, all the classes grow in the same way, giving the less represented class similar importance as the dominant class. Even if enough data are generated, the classes remain imbalanced because CSBS considers component size for oversampling the instances, which can vary significantly. Therefore, the next step consists of resampling the new obtained set by applying random undersampling to remove any new dominance of an oversampled class. The main steps of CSBS are summarized in Algorithm 2.

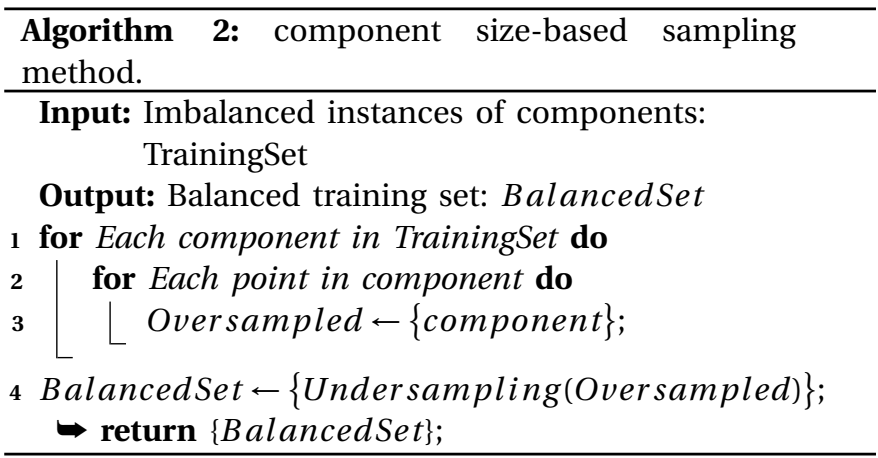

\section{STUDY AREA AND DATA PREPARATION}

\section{A. Study Area}

The performance of the proposed approach is assessed using ALS point cloud provided by the German Society for Photogrammetry, Remote Sensing, and Geoinformation (DGPF) [55]. It was acquired on 21 August 2008 by Leica Geosystems, using a Leica ALS50 system, with a $45^{\circ}$ field of view and a mean flying height of $500 \mathrm{~m}$ above ground. The ALS data used is characterized by a low density (46 points $/ \mathrm{m}^{2}$ ). It is covering an urban area located in residential parts of Vaihingen city in southern Germany. It is featured by an abundance of different types of roof superstructures.Fig. 4 shows the three studied types of building roof superstructures. The study area covers 1,577 buildings and contains 2,810,723 points. The application of subsurface growing assigned 2,703,901 points to segments (Fig. 5 (b)), and ignored 106,822 points (Fig. 5 (a)). The unassigned points represent the proposed method's starting 
TABLE I: Features used for components classification.

\begin{tabular}{|c|c|c|}
\hline Features & Designation & Definition \\
\hline Feature 1 & $\bar{N}$ & size of component \\
\hline Feature 2 & $\Delta Z$ & $=Z_{\max }-Z_{\min }$ \\
\hline Feature 3 & $\Delta \operatorname{Max}(Z)_{\text {building/component }}$ & $=Z_{\text {max in building }}-Z_{\text {max in component }}$ \\
\hline Feature 4 & Area $_{(\text {convex-hull) }}$ & \multirow{2}{*}{ Shoelace formula for polygon area } \\
\hline Feature 5 & $\operatorname{Area}_{(\alpha-\operatorname{shape~})}$ & \\
\hline Feature 6 & $Z_{\min }$ & $\min (Z)$ in component \\
\hline Feature 7 & $Z_{\max }$ & $\max (Z)$ in component \\
\hline Feature 8 & $\mu_{Z}$ & $\mu(Z)$ of component \\
\hline Feature 9 & $I_{\text {size }}($ component $)$ & $=-\log _{2} \frac{\text { size }(\text { component })}{\text { size(building })}$ \\
\hline Feature 10 & Entropy $(Z)$ & $=-\sum_{i=1}^{N}\left(\frac{Z_{p i}}{\sum_{j=1}^{N} Z_{p j}} \log _{2} \frac{Z_{p i}}{\sum_{j=1}^{N} Z_{p j}}\right.$ \\
\hline Feature 11 & $\sigma_{Z}$ & $=\sqrt{\frac{1}{N} \sum_{i=1}^{N} Z_{i}^{2}-\overline{\mu_{Z}^{2}}}$ \\
\hline Feature 12 & $C_{v}$ & $=\frac{\sigma_{Z}(\text { component })}{\mu_{Z}(\text { component })}$ \\
\hline
\end{tabular}

point to reassign the points to their corresponding classes, namely shed and gable dormers, ground, chimneys, and others.

\section{B. Data Preparation and Parameters Tuning}

The orthophoto is illustrated in Fig. 6 with two distinct parts that contain 25695 points belonging to 552 buildings selected for learning the classifier. The two subsets are merged into a single data set to make instances more representative. The metric considered for connected components is Euclidean distance $=1.5 \mathrm{~m}$. The distance is defined considering the targeted classes and the point cloud density. Typical dormers and chimneys measure not less than $1 \mathrm{~m}$. Furthermore, considering a larger distance, for example, equal or greater than $2 \mathrm{~m}$, could generate one component of two nearly distinct small objects like dormers, chimneys, or antennas. Thus, by taking into account the density of the point cloud, which is around 4-6 points $/ \mathrm{m}^{2}$, an average of $1.5 \mathrm{~m}$ is taken as a reference distance. Only components having five points and more are retained. For each resulting component, twelve features are computed (listed in Table I). The used alpha shape radius is $1.5 \mathrm{~m}$. The training subset, representing $70 \%$ of the labeled data set, is randomly picked, and the remaining samples are used for testing. For classification, the samples were normalized in $[0,1]$. For the SVM classifier, the radial basis function (RBF) kernel is used. RBF kernel with an appropriate selection of parameters was successfully adopted in several papers [39], [56]. Parameter selection consists of using grid search and 5-fold cross-validation to determine hyperparameters giving the best classification results. For SVM, grid search is to varying the cost parameter $C$ in $\left\{2^{-3}, 2^{-1}, 2^{1}, 2^{3}, 2^{5}, \ldots 2^{15}\right\}$ and $\gamma$ RBF kernel parameter in $\left\{2^{-15}, 2^{-13}, . .2^{-1}, 2^{1}, 2^{3}\right\} .5$ fold cross-validation is to divide the training subset into 5 -folds, then each fold of the subset is tested once while
4 remaining folds are used to train the classifier. Crossvalidation permits to prevent overfitting. The parameters of Random Forest classifier are n_estimators the number of trees in the forest, and criterion \{"gini", "entropy"\} the function to measure the quality of a split. This last is the parameter also of the decision tree classifier and max_depth the maximum depth of the tree. For AdaBoost, the Decision Tree is used as the base estimator from which the boosted ensemble is built, and n_estimators is the maximum number of estimators at which boosting is terminated. For all the classifiers, parameters selection is performed in the same way described above for SVM.

\section{RESUltS AND DISCUSSION}

\section{A. Connected Components Analysis Results}

The unassigned points are subjected to the connected component analysis stage. Fig. 7 presents the resulting components. Components belonging to the same building are differently colored to differentiate the diverse objects, which is useful when labeling them. Starting from 106,833 points, the process generated 15,817 components (Fig. 7 (a)). The components with less than five points are not considered, which led to 4,067 components (Fig. 7 (b)). The neighboring distance is empirically chosen, considering the point cloud density (4 to $6 \mathrm{point} / \mathrm{m}^{2}$ ) and the particularity of the roof superstructures detection problem. In general, the distance between any two points belonging to two different dormers or chimneys should not exceed $1.5 \mathrm{~m}$. Taking a smaller measure leads to an unrepresentative component, while considering a larger measure can lead to a mega component containing several objects. Some examples of resulting components projected over the orthophoto are shown in Fig. 8. The choice of measurement and metric directly affects the generated components. Fig. 9 shows two shed dormers (Fig. 9a) and two gable dormers (Fig. 9b) 


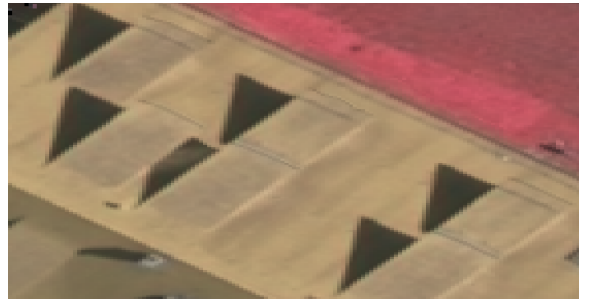

(a)

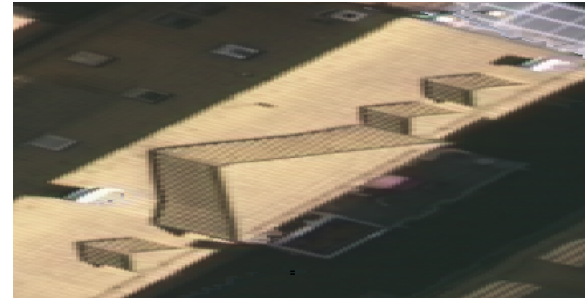

(b)

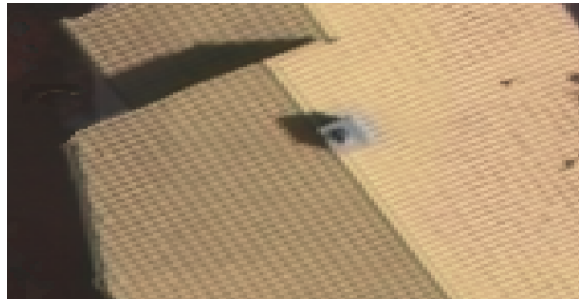

(c)

Fig. 4: Types of studied building roof superstructures. (a) Shed dormer. (b) Gable dormer. (c) Chimney.

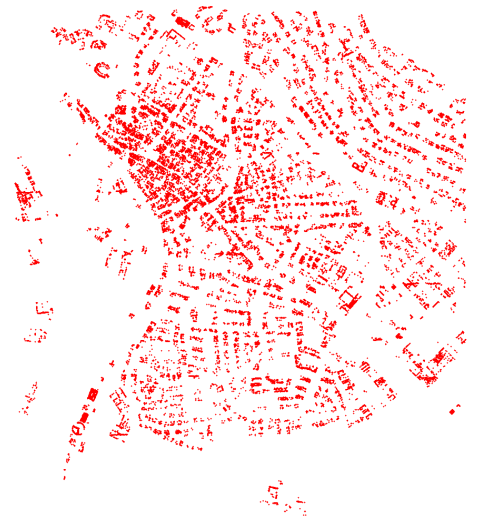

(a)

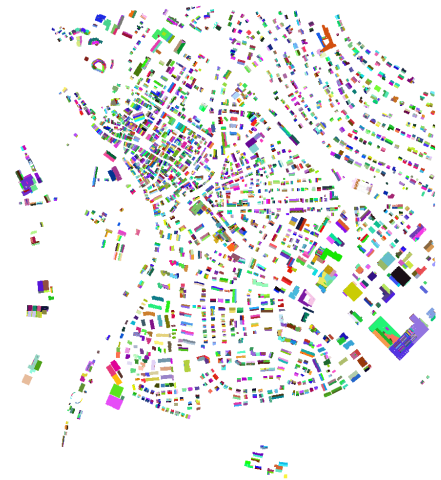

(b)

Fig. 5: Results from subsurface growing. (a) Unassigned points (in red). (b) Points assigned to segments.

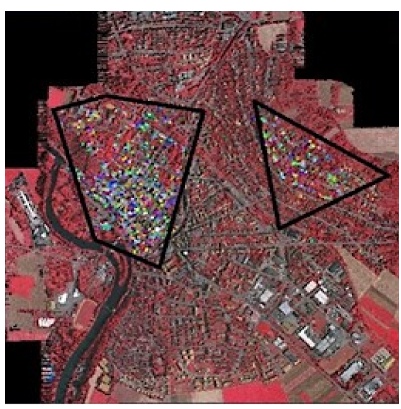

Fig. 6: The parts of region forming the labeled data set.

considered as one component due to their close proximity. There is no standard distance metric valid for all targeted

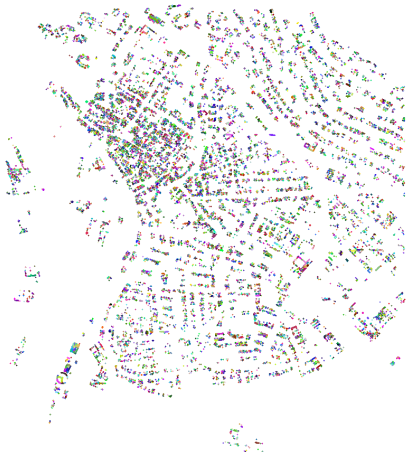

(a)

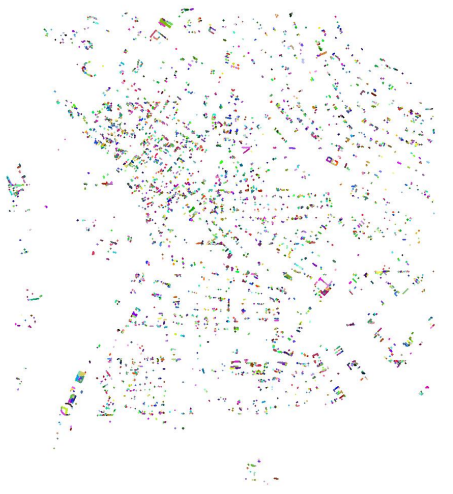

(b)

Fig. 7: results of the connected component analysis. (a) 15,817 resulting components. (b) 4,067 resulting components after filtering components with less than five points.

classes. Several metrics and measures can be tested; however, this is very costly as a new interactive labeling stage is required, which can be seen as a drawback of the presented method.

\section{B. Interactif Labeling}

The orthophoto of the studied area, illustrated in Fig. 6, shows two selected regions serving to form the labeled data set. It contains 25,695 points forming 1,412 components shown in Fig. 10. For classification results evaluation, the data set is labeled by projecting the components over the orthophoto that served as ground truth. The components are labeled one by one by verifying the class on which the 


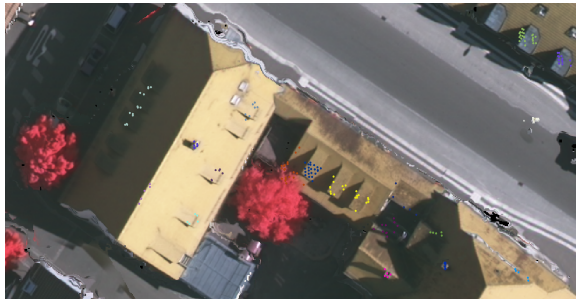

(a)

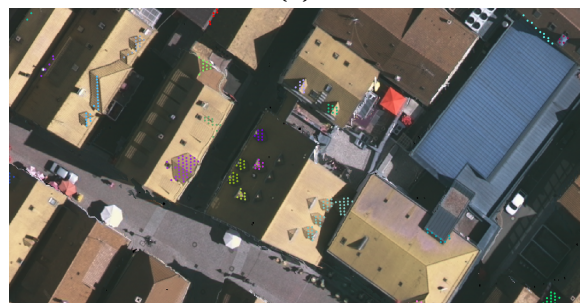

(d)

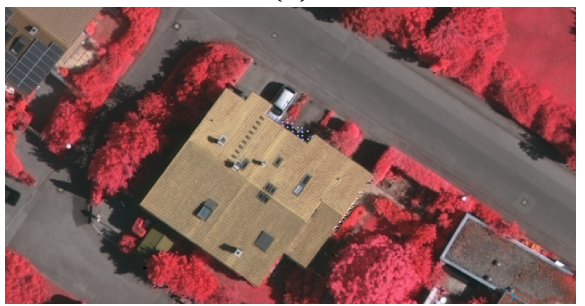

(g)

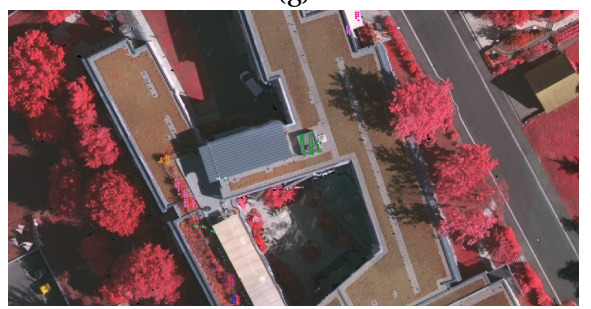

(j)

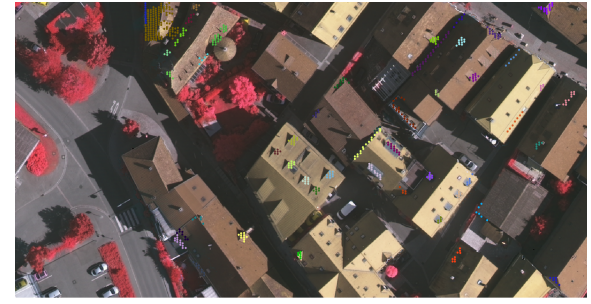

(b)

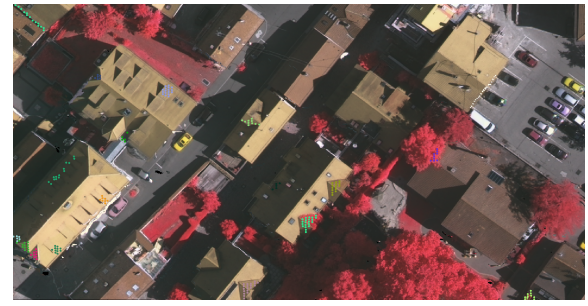

(e)

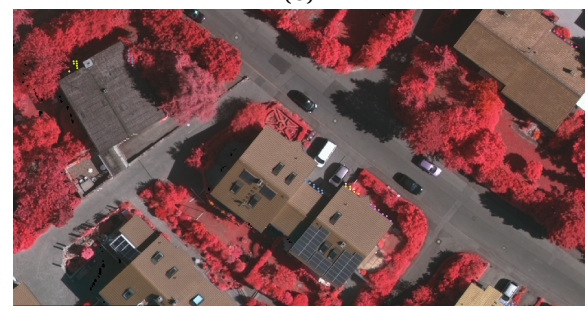

(h)

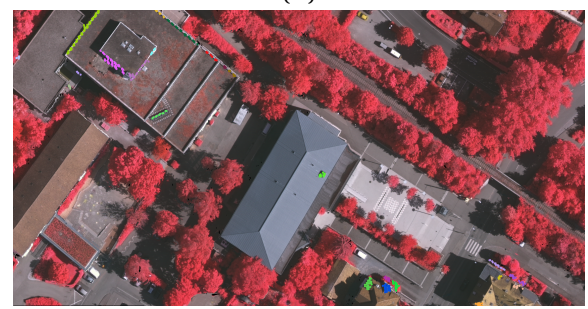

(k)

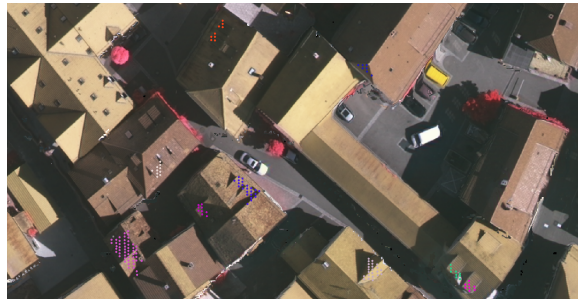

(c)

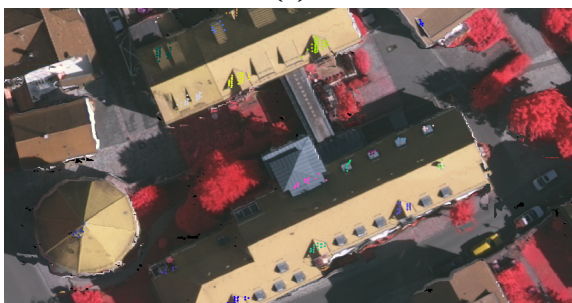

(f)

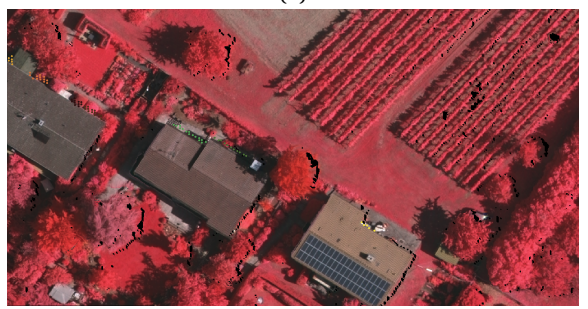

(i)

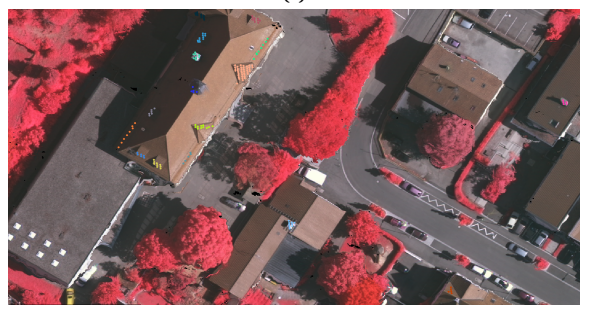

(l)

Fig. 8: Projection of the resulting components over the orthophoto. (a),(b),(c) Shed dormer. (d),(e),(f) Gable dormer. (g),(h),(i) Ground. (j),(k),(l) Chimney components.

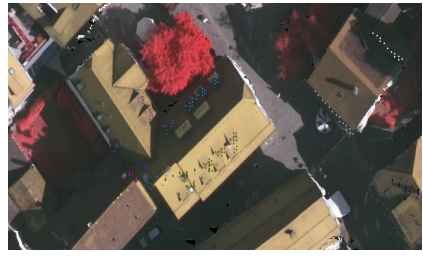

(a)

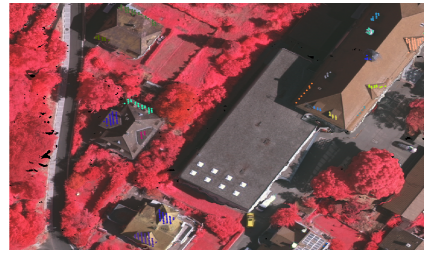

(b)

Fig. 9: Roof dormer components projected over a part of orthophoto. Resulting component considering: (a) (02) shed dormers as a single component; (b) (02) gable dormers as a single component.

component points coincide from the orthophoto. For each component, a unique number is assigned. The same value is also assigned to the points forming that component. Table II illustrates the detail of each of the five studied classes. The fifth class named 'others' represents objects, such as vegetation, car, facade, and any objects except shed

and gable dormers, ground, and chimneys.
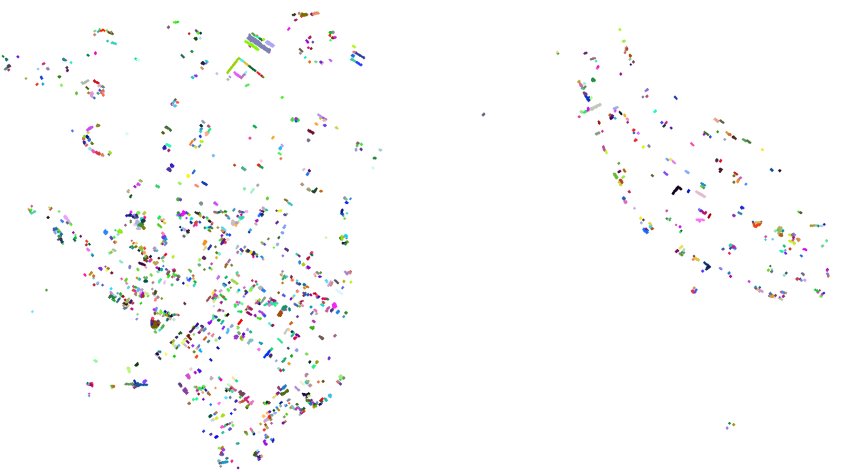

Fig. 10: The resulting components using connected component analysis from the selected samples. 
TABLE II: Detail of each class from selected samples regions.

\begin{tabular}{lccc}
\hline \hline Classes & Designation & Number of components & Total number of points \\
\hline Class 1 & shed dormer & 241 & 3815 \\
class 2 & gable dormer & 100 & 2178 \\
class 3 & ground & 47 & 834 \\
class 4 & chimney & 155 & 1235 \\
class 5 & Others & 869 & 17633 \\
\hline \hline
\end{tabular}

\section{Classification Results}

\section{1) Evaluations Metrics:}

In addition to overall accuracy (OA) and execution time, the effectiveness metrics include recall, precision, F-score, and area under the curve (AUC) score. The recall is the ratio between true positive (TP) and the sum of TP and false negative (FN). Precision is the ratio between true positive (TP) and the sum of TP and false positive (FP). F-score expresses the balance between the precision and the recall [57]. Parameters selection is evaluated using F1score because accuracy is not suited for imbalanced data, due to the influence of the majority class that increases the final accuracy score.

\section{2) Classification of Original Data:}

A partitioning example of training and testing subsets is shown in Table III, representing respectively $70 \%$ and $30 \%$ from the labeled samples set. It is worth mentioning that the data are highly imbalanced. The class 'others' is the most predominant class with 869 instances, followed by shed dormer with 241 samples and chimneys coming third with 155 components and gable dormers with 100 samples. The ground class is the less represented class with 47 components. This has a significant influence on the SVM classification results, as shown in Table IV because the SVM classifier is affected by the classes imbalance issue [58]. The accuracy $(59.67 \%)$ is not representative as a high score is usually obtained through classifying all observations as the majority class.

\section{3) Classification of Resampled Data:}

Imbalanced samples lead necessarily to overfitting where all the samples are trained as the class 'others'. In order to rebalance the classes, different resampling methods are tested. Table $\mathrm{V}$ shows the new partition of data sets for classification purposes.

\section{a) Random Undersampling of Train Subset}

Training data are formed by randomly undersampling the training subset based on the least represented class (i.e., ground). Table VI presents the confusion matrix of SVM classification of undersampled data. The weakness of true positive scores and global accuracy is due to the few numbers of the training set (165) representing less than $15 \%$ of all samples (1412). Random undersampling is not appropriated, as it tends to reduce the samples, whereas a higher number of training data is needed.

\section{b) Random Oversampling of Train Subset}

Training data are formed by random oversampling training subset based on the most represented class (i.e., 'others'). The analysis of the SVM classification results from Table VII shows a minor improvement in the F1-score of the training set, OA and AUC. Except for the dominant class 'others', the TP scores remain insufficient for most classes. The main confusions are recorded with the 'others' class. The method duplicates training instances, which generates not enough representative subset of the original classes (i.e., lack of representative samples).

\section{c) Component Size-Based Sampling Method}

TThe components are oversampled based on their size. Each component is duplicated according to the number of points it contains. Then, to rebalance the classes, random undersampling is performed on the obtained set. Table VIII illustrates the new distribution of data. Table IX shows the SVM classification results of resampled data by CSBS. Using the training subset, F1-score is close to $86 \%$. For the test subset, the obtained OA exceeds $87 \%$, AUC 0.96 , and the processing time 10 seconds. F1-score is high for all the classes. For the dominant class 'others', F1-score reached $91 \%$, and $88 \%$ for the ground class, while all the ground components are correctly classified, which is a good performance considering the smallest class. The misclassification of some class "others" components reduces the precision rates of the classes shed and gable dormers, ground, and chimney. This is due to the heterogeneous nature of the class 'others', which includes objects, such as trees, electric wires, and antennas. These objects can be confused with chimneys as they are situated on a building roof. An object like a car or bicycle can also be interpreted as the ground, considering the low density of the point cloud. The SVM classification of resampled data using CSBS is performed a second time, starting from a new splitting of data set with similar distribution with the previous data set (table VIII). Again, the results achieved high scores (Table IX). Similar performance measurements (recall, precision, and F1-score) are noted for all the classes, except for the chimneys class, for which the precision slightly decreased to $56 \%$, and that for shed dormer, which increased to $77 \%$.

\section{d) Synthetic Resampling Methods}

Different synthetic sampling methods are applied. Table X illustrates the partitioning of the data set according to each synthetic method. The data are split up in train and test subsets in the same way as described previously and submitted to SVM classifier. The classification results are depicted in Table XI. The obtained (TP) via the different 
TABLE III: Partition of training and testing subsets representing respectively $70 \%$ and $30 \%$ from the labeled samples set.

\begin{tabular}{lccc}
\hline \hline Classes & Train subset(70\%) & Test subset (30\%) & Total instances by class \\
\hline shed dormer & 169 & 72 & 241 \\
gable dormer & 70 & 30 & 100 \\
ground & $\mathbf{3 3}$ & 14 & 47 \\
chimney & 108 & 47 & 155 \\
Others & 608 & 261 & 869 \\
\hline Total & 988 & 424 & 1412 \\
\hline \hline
\end{tabular}

TABLE IV: Confusion matrix and metrics resulting from SVM classification of raw imbalanced data $(C=32768, \sigma=1$, training $F 1-$ score $=32.32 \%)$

\begin{tabular}{lccccc}
\hline \hline Classes & Shed & Gable & Ground & Chimney & Others \\
\hline Shed & $\mathbf{2 4}$ & 5 & 2 & 0 & 41 \\
Gable & 2 & $\mathbf{8}$ & 0 & 0 & 20 \\
Ground & 1 & 1 & $\mathbf{4}$ & 0 & 8 \\
Chimney & 8 & 3 & 1 & $\mathbf{1 1}$ & 24 \\
Others & 29 & 6 & 5 & 15 & $\mathbf{2 0 6}$ \\
\hline OA & \multicolumn{5}{c}{$\mathbf{5 9 . 6 7} \mathbf{0 . 7 5}$} \\
\hline AUC & \multicolumn{6}{c}{$\mathbf{6 4 . 0 8}$ s } \\
\hline Time &
\end{tabular}

methods can not be compared because the number of instances varies depending on the used method. The SMOTETOMEK produced the best AUC (0.96) and third best time processing $(22.51 \mathrm{~s})$ after KMEANSSMOTE (14.06 s) and SVMSMOTE (18 s). This last gives the lower OA (85.25\%). BORDERLINESMOTE achieved to the best OA (89.41\%) exceeding KMEANSSMOTE OA (88.81\%) and ADAYSN OA (88.31\%), but with a smaller AUC (0.93). Classification of data generated by SMOTE method takes the longest time (83 s) and gives the smallest AUC with (0.93), even if the difference is small compared to the AUC of the other methods. The best F1-score for gable dormer and chimney classes are recorded by ADAYSN with $94 \%$ and $91 \%$ respectively. BORDERLINESMOTE produces the highest F1score for shed dormer $(88 \%)$ and ground $(99 \%)$ followed by SMOTETOMEK and ADASYN giving respectively (87\%), $(86 \%)$ for shed dormer and $(94 \%)$, for gable class, which is a small difference. Taking the AUC scores and the time execution as criteria, the most efficient synthetic technique is SMOTETOMEK followed by ADASYN, KMEANSSMOTE, BORDERLINESMOTE, SVMSMOTE and SMOTE.

\section{e) Sampling Method Comparison}

CSBS method is compared with SMOTE, KMEANSSMOTE, SMOTETOMEK, ADASYN and BORDERLINESMOTE, the studied methods in previous section. From Tables IX and XI, CSBS is the faster regarding the processing time with 10.03 s, while SMOTETOMEK took $22.51 \mathrm{~s}$, ADASYN (29.75 s) and BORDERLINESMOTE with $52.5 \mathrm{~s}$. CSBS and SMOTETOMEK give the best AUC with 0.96 . For the other methods, the AUC is close to 0.94 . The accuracy reached $87.97 \%$ for CSBS, close to the highest accuracy produced by BORDERLINES-
MOTE (89.41\%). The Fig. 11. shows multiclass Receiver Operating Characteristic Curve for the the most powerful methods. Classes shed and gable dormers, ground and chimney AUC scores exceeds 0.90 for all methods. For the class 'others', the best AUC is obtained with the proposed method (0.90) which is a good score considering the heterogeneous nature of the class. It has reached 0.88 for SMOTETOMEK and 0.81 for KMEANSSMOTE. However, it does not exceed 0.79 for ADAYSN and BRODERLINESMOTE. Fig. 12 presents a comparison between F1-scores of methods. The results show that ADASYN and BORDELINESMOTE give the best results in term of F1-score for majority of classes except for 'others' class. With SMOTETOMEK, KMEANSSMOTE, ADASYN and BORDERLINESMOTE, precisions of classes are higher than CSBS except for 'others' class, which means that false positive rates are low. Inversely, CSBS leads to a higher recall for all classes than the other methods, due to weak of false negatives values especially for the 'others' class. The low density of the point cloud and the arrangement of points within the components cause the misclassification in some cases. The distinction between each other is difficult, because there is a lot of types of roof superstructures. There are so many that sometimes they end up looking alike. Some confusions between shed dormer and chimney classes are observed for all the methods. Chimney may be confused with shed dormer when it is not located on the top roof building. Chimneys and dormers can share similar shapes, especially when the components contain few points in their upper part. None of the twelve features used is able to discriminate this specific case. The bigger the point cloud density, the more accurate are features, and the higher are the classification performance measurements. The use of sampling techniques requires some rules. Yokoya et al. [59] used a fusion of images and data vectors for local climate zone classification. The data used were very heterogeneous within the train set but also inter-training and test subsets. Consequently, the data resampling did not enhance the classification. In the present study, the data used are homogeneous. From point cloud covering one region, the split in train and test subsets is done randomly.

\section{f) Features Analysis}

The correlation matrix of the proposed features is depicted in Fig. 13. One can see that some features are highly correlated, such as features 6,7 , and 8 , based on component height information. A strong correlation is also recorded between features 2,11 , and 12 , representing the point 
TABLE V: DETAILS OF TRAINING AND TESTING SUBSETS USING UNDERSAMPLING BASED ON THE LEAST REPRESENTED CLASS WITH 33 COMPONENTS AND OVERSAMPLING BASED ON THE MOST REPRESENTED CLASS WITH 608 COMPONENTS)

\begin{tabular}{lcccc}
\hline \hline Classes & Original train subset & Undersampled train subset & Oversampled train subset & Test subset \\
\hline Shed & 169 & 33 & 608 & 72 \\
Gable & 70 & 33 & 608 & 30 \\
Ground & $\mathbf{3 3}$ & 33 & 608 & 14 \\
Chimney & 108 & 33 & 608 & 47 \\
Others & $\mathbf{6 0 8}$ & 33 & 608 & 261 \\
\hline Total & 988 & 165 & 3040 & 424 \\
\hline \hline
\end{tabular}

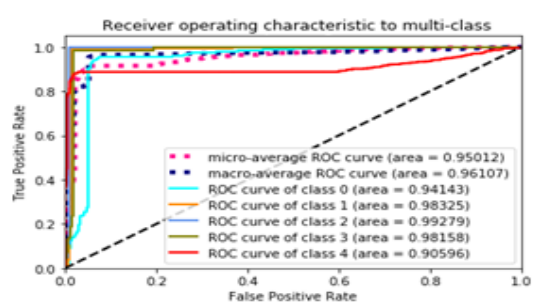

(a)

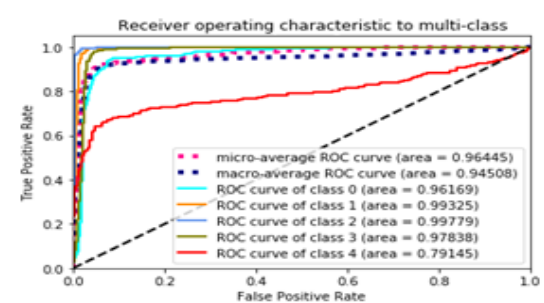

(b)

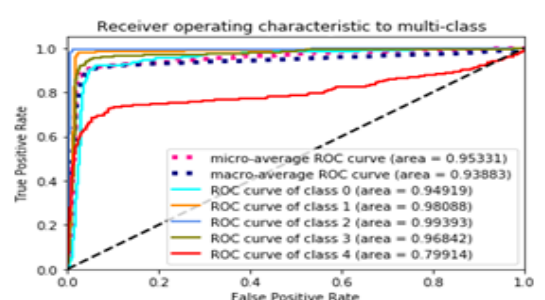

(c)

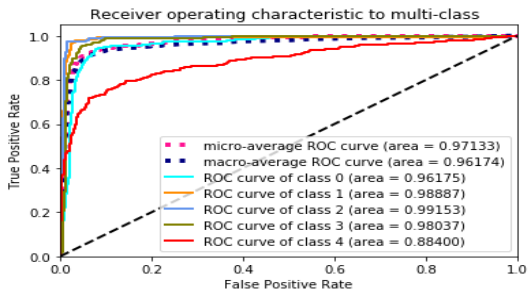

(d)

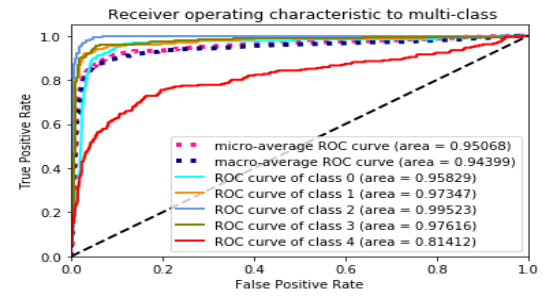

(e)

Fig. 11: Receiver Operating Characteristic Curve: (a) CSBS. (b) ADASYN. (c) BORDERLINESMOTE. (d) SMOTETOMEK. (e) KMEANSSMOTE. Class 0: Shed dormer, class 1: Gable dormer, class 2: Ground, class 3: Chimney, class 4: Others.

\section{TABLE VI: CONFUSION MATRIX AND METRICS RESULTING FROM SVM CLASSIFICATION OF UNDERSAMPLED TRAINING SUBSET \\ $(C=0.03125, \sigma=0.00781$, training $F 1-$ score $=0.0 \%)$}

\begin{tabular}{lccccc}
\hline \hline Classes & Shed & Gable & Ground & Chimney & Others \\
\hline Shed & $\mathbf{8}$ & 0 & 40 & 23 & 1 \\
Gable & 2 & $\mathbf{0}$ & 23 & 4 & 1 \\
Ground & 1 & 0 & $\mathbf{1 3}$ & 0 & 0 \\
Chimney & 0 & 2 & 37 & 7 & 1 \\
Others & 26 & 4 & 184 & 37 & $\mathbf{1 0}$ \\
\hline OA & \multicolumn{5}{c}{$\mathbf{0 0 . 0 9}$ \% } \\
\hline AUC & $\mathbf{0 . 5 5}$ \\
\hline Time & $\mathbf{0 . 0 1} \mathbf{~ s}$ \\
\hline \hline
\end{tabular}

height variation in the component (table I). Features are selected based on Pearson correlation values shown in table XII. Features strongly correlated with values greater than 0.9 are removed. (i.e., features $7,8,11,12$ are removed). Only features 1, 2, 3, 4, 5, 6, 9, 10 will be considered in future tests.

g) Comparaison of Different Classifiers

A comparison of the most used classifiers for the ALS point

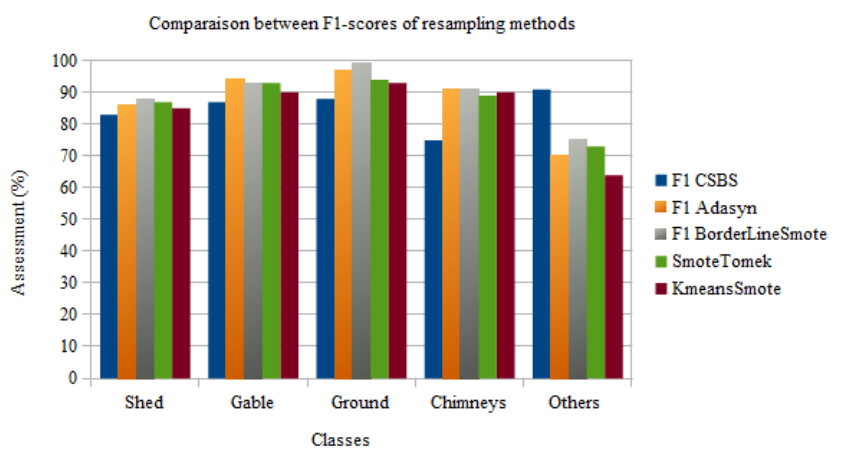

Fig. 12: Comparison between F1-score of methods.

cloud is done from the resampled data using the methods studied previously (CSBS, ADAYSN, BORDERLINESMOTE, SMOTE, SVMSMOTE, KMEANSSMOTE, SMOTETOMEK). In addition to SVM [58], [60], the concerned classifiers are Random Forests (RF) [61]-[63], Decision Tree (DT) [64], [65] and AdaBoost [49], [60]. The best eight selected features are used. Table XIII illustrates the obtained results. SVM is the unique classifier giving good results for the seven sampling methods used among the four tested classifiers. The ob- 


\section{TABLE VII: CONFUSION MATRIX AND METRICS RESULTING FROM SVM CLASSIFICATION OF OVERSAMPLED TRAINING SUBSET \\ $(C=2048, \sigma=8$, training $F 1-$ score $=50.66 \%)$}

\begin{tabular}{lccccc}
\hline \hline Classes & Shed & Gable & Ground & Chimney & Others \\
\hline Shed & $\mathbf{2 3}$ & 5 & 2 & 7 & 35 \\
Gable & 5 & $\mathbf{1 0}$ & 0 & 2 & 13 \\
Ground & 3 & 0 & $\mathbf{3}$ & 2 & 6 \\
Chimney & 6 & 2 & 4 & $\mathbf{9}$ & 26 \\
Others & 34 & 7 & 8 & 20 & $\mathbf{1 9 2}$ \\
\hline OA & \multicolumn{5}{|c}{$\mathbf{5 5 . 8 9}$ \% } \\
AUC & $\mathbf{0 . 7 1}$ \\
\hline Time & $\mathbf{2 2 . 4 3} \mathbf{~ s}$ \\
\hline \hline
\end{tabular}

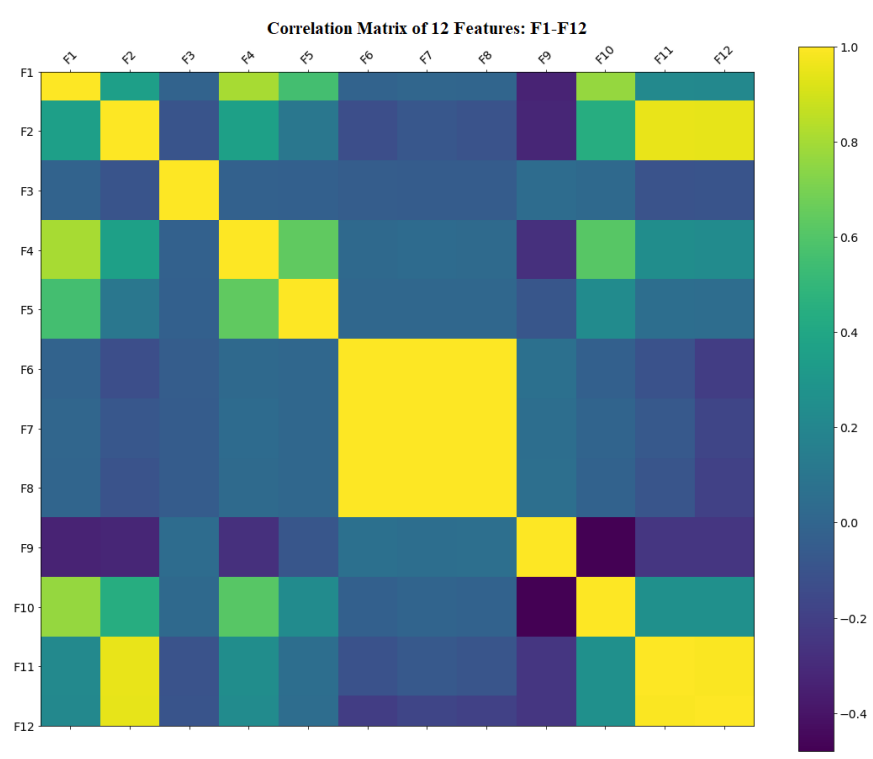

Fig. 13: The correlation matrix of all the used features: F1-F12

tained results using the eight selected features are almost similar to those previously obtained with twelve features particularly using CSBS. The best results are recorded with CSBS for the classifiers SVM, RF and DT. SVM gave the best performance with OA (88.28\%) and AUC (0.96). RF and DT classifiers achieved to a good competitive performance with SVM, where OA reached $85.93 \%, 87.21 \%$ and AUC to $0.96,0.95$ respectively. The best time processing is obtained with a decision tree with $(0.02 \mathrm{~s}), \mathrm{RF}(2.17 \mathrm{~s})$, and SVM with 9.9 s. Like SVM, RF and DT classifiers are well suited for small objects such as cars, trees, power lines, curbs, barriers, and artifacts poles [65]-[67] which explain the good performance obtained in the present context. Lower scores are obtained with the AdaBoost classifier for all the sampling methods tested. Compared to [49], [68], [69], AdaBoost was successfully used for ALS point cloud classification AdaBoost classifier does not perform well for the targeted classes. In [60], similar weak accuracy is produced by AdaBoost classifier for Pole-like objects. A major drawback of the SVM classifier is to disregard the spatial correlation in class labeling [14], [49]; for example, a distinct point can be classified as a building even if all surrounding points are classified as vegetation. In this study, a segment-based classification is considered rather than point-based, where the segments (i.e., components) are formed by connected components analysis, and then the features are computed for each component rather than for every point. The Gaussian kernel associated with selecting the best parameters using grid search and cross validation [70] allows SVM to outperform the RF classifier, known to be one of the best classifiers [17]. The use of more sophisticated kernels does not necessarily improve the results [58]. A similar segmentbased approach was proposed in [39] for SVM classification of segmented ALS point clouds in urban areas. However, the authors considered dormers and chimneys as micro-objects that lead to misclassifications. Thus, roof superstructures are post-processed by classifying them into nearby larger segments, which means classifying them as buildings.

\section{CONCLUSION}

This paper presented two major contributions for building roof superstructures classification from imbalanced and low-density airborne LiDAR point clouds. A classification approach is proposed to discriminate nonplanar surfaces, including building roof superstructures and the imbalanced classes in LiDAR point cloud data. Also, connected component analysis is introduced for clustering nonplanar surfaces from a low-density ALS point cloud by defining only a neighborhood distance as criteria. Twelve geometricbased features are computed from the resulting components and used as input by the classification procedure. Here, five classes with imbalanced samples have been considered: shed and gable dormers, chimneys, ground, and others. A resampling strategy has been investigated to remove the dominance of classes. Specifically, CSBS has been introduced, and other synthetics sampling methods used in literature are applied (i.e., SMOTE, BORDERLINESMOTE, SVMSMOTE, KMEANSSMOTE, SMOTETOMEK, and ADASYN). After using the SVM classifier and parameters selection, the synthetics methods giving the highest performances are compared with CSBS. Results show that the classification of CSBS data reduces false-negative rates, especially for the dominant class, while for KMEANSSMOTE, SMOTETOMEK, ADASYN and BORDERLINESMOTE data, the false-positive rates are reduced excepting for the 'others' class. The most used classifiers for ALS data are compared based on the best eight selected features. The obtained classification results are satisfactory considering the sparse data used. Despite the small-sized data and their imbalanced nature, suitable performances have been achieved. In contrast to images, where free and big data sets are available, like ImageNet, the lack of labeled airborne LiDAR data sets remains a substantial problem so far. According to targeted classes, instances labeling is a tedious, timeconsuming, and costly task in itself. The present method leads the way to future studies to address the lack of training samples and difficulties in preparing training data 
TABLE VIII: DETAILS OF TRAINING AND TESTING SUBSETS OBTAINED BY CSBS METHOD

\begin{tabular}{lccccc}
\hline \hline Classes & Components & Points & training subset & New training subset & Test subset \\
\hline Shed & 241 & 3815 & 2670 & 584 & 1145 \\
Gable & 100 & 2178 & 1525 & 584 & 653 \\
Ground & 47 & 834 & $\mathbf{5 8 4}$ & 584 & 250 \\
Chimney & 155 & 1235 & 864 & 584 & 371 \\
Others & 869 & 17633 & 12343 & 584 & 5290 \\
\hline Total & 1412 & 25695 & 17986 & 584 & 7709 \\
\hline \hline
\end{tabular}

TABLE IX: CONFUSION MATRIX AND METRICS RESULTING FROM SVM CLASSIFICATION OF DATA RESAMPLED BY CSBS

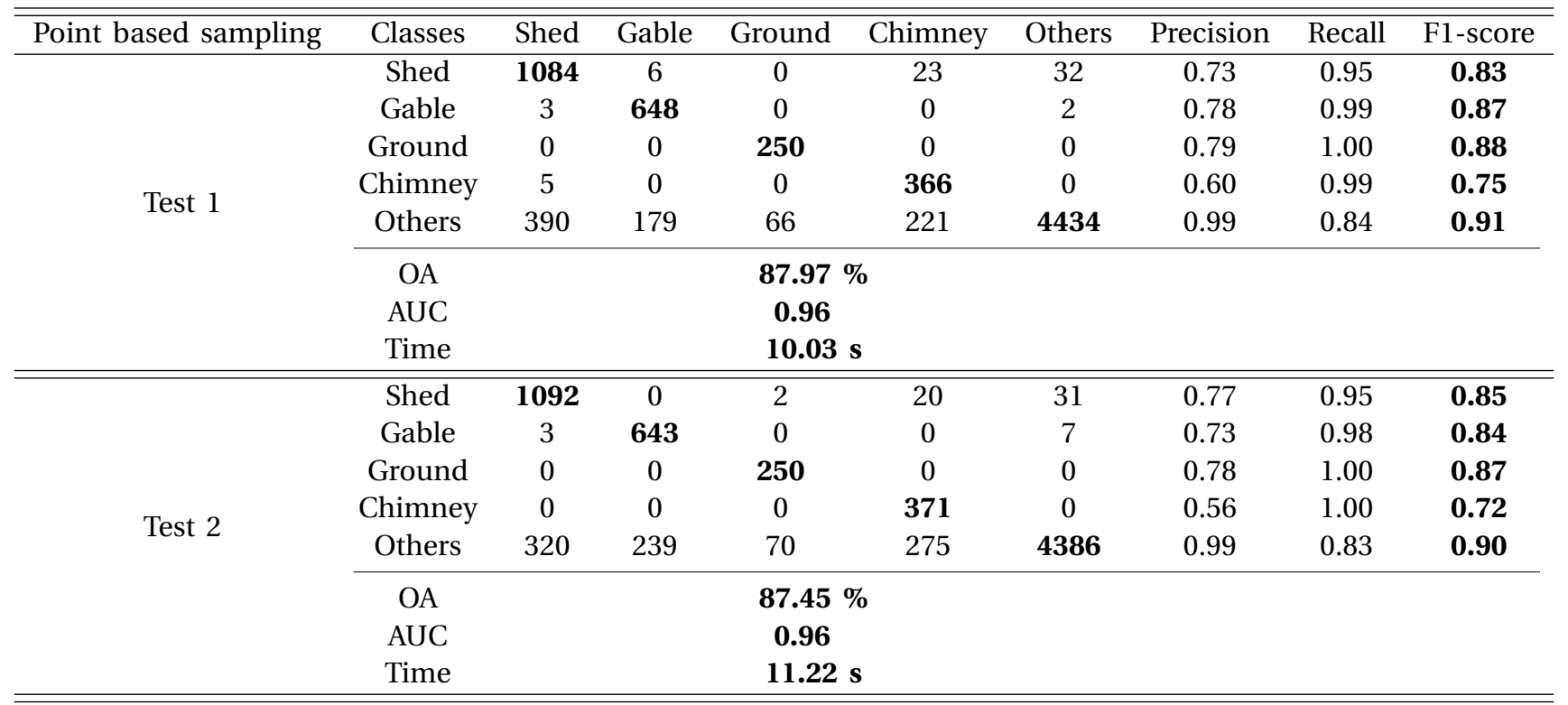

TABLE X: Partition of training set according to each synthetic method

\begin{tabular}{lccccccc}
\hline \hline Classes & $\begin{array}{c}\text { Original } \\
\text { data set }\end{array}$ & $\begin{array}{c}\text { Smote } \\
\text { data set }\end{array}$ & $\begin{array}{c}\text { BorderLineSmote } \\
\text { data set }\end{array}$ & $\begin{array}{c}\text { SVMSmote } \\
\text { data set }\end{array}$ & $\begin{array}{c}\text { Adasyn } \\
\text { data set }\end{array}$ & $\begin{array}{c}\text { KmeansSmote } \\
\text { data set }\end{array}$ & $\begin{array}{c}\text { SmoteTomek } \\
\text { data set }\end{array}$ \\
\hline Shed & 241 & 869 & 869 & 869 & 870 & 869 & 848 \\
Gable & 100 & 869 & 869 & 869 & 844 & 869 & 841 \\
Ground & 47 & 869 & 869 & 425 & 887 & 869 & 861 \\
Chimney & 155 & 869 & 869 & 869 & 893 & 869 & 856 \\
Others & 869 & 869 & 869 & 869 & 869 & 869 & 817 \\
\hline Total & 1412 & 4345 & 4345 & 4345 & 4363 & 4345 & 4223 \\
\hline \hline
\end{tabular}

usable to train any classifier. Also, this research may be integrated into the framework for 3D reconstruction of complex buildings to give a realistic final model as it permits to restore roof elements, hitherto omitted. Alternatively, deep learning techniques could be investigated to automatically extract more complex roof details, such as hipped dormers or antennas.

\section{ACKNOWLEDGMENT}

The Vaihingen data set was provided by the German Society for Photogrammetry, Remote Sensing and Geoinformation (DGPF) [55]. This research was conducted in the Technische Universität (TU) Berlin. The authors would like to thank TU Berlin for support and publication funding.

\section{REFERENCES}

[1] C. Zhao, H. Guo, J. Lu, D. Yu, D. Li, and X. Chen, "Als point cloud classification with small training data set based on transfer learning," IEEE Geoscience and Remote Sensing Letters, 2019.

[2] A. Adán, T. Prado, S. Prieto, and B. Quintana, "Fusion of thermal imagery and lidar data for generating tbim models," in 2017 IEEE SENSORS. IEEE, 2017, pp. 1-3.

[3] M. Li, F. Rottensteiner, and C. Heipke, "Modelling of buildings from aerial lidar point clouds using tins and label maps," ISPRS Journal of Photogrammetry and Remote Sensing, vol. 154, pp. 127-138, 2019.

[4] Y. Wang, J. Hyyppä, X. Liang, H. Kaartinen, X. Yu, E. Lindberg, J. Holmgren, Y. Qin, C. Mallet, A. Ferraz et al., "International benchmarking of the individual tree detection methods for modeling 3-d canopy structure for silviculture and forest ecology using airborne laser scanning," IEEE Transactions on Geoscience and Remote Sensing, vol. 54, no. 9, pp. 5011-5027, 2016.

[5] W. Dai, B. Yang, Z. Dong, and A. Shaker, "A new method for 3d individual tree extraction using multispectral airborne lidar point 
TABLE XI: CONFUSION MATRIX AND METRICS RESULTING FROM THE SVM CLASSIFICATION OF DATA RESAMPLED USING SYNTHETIC METHODS

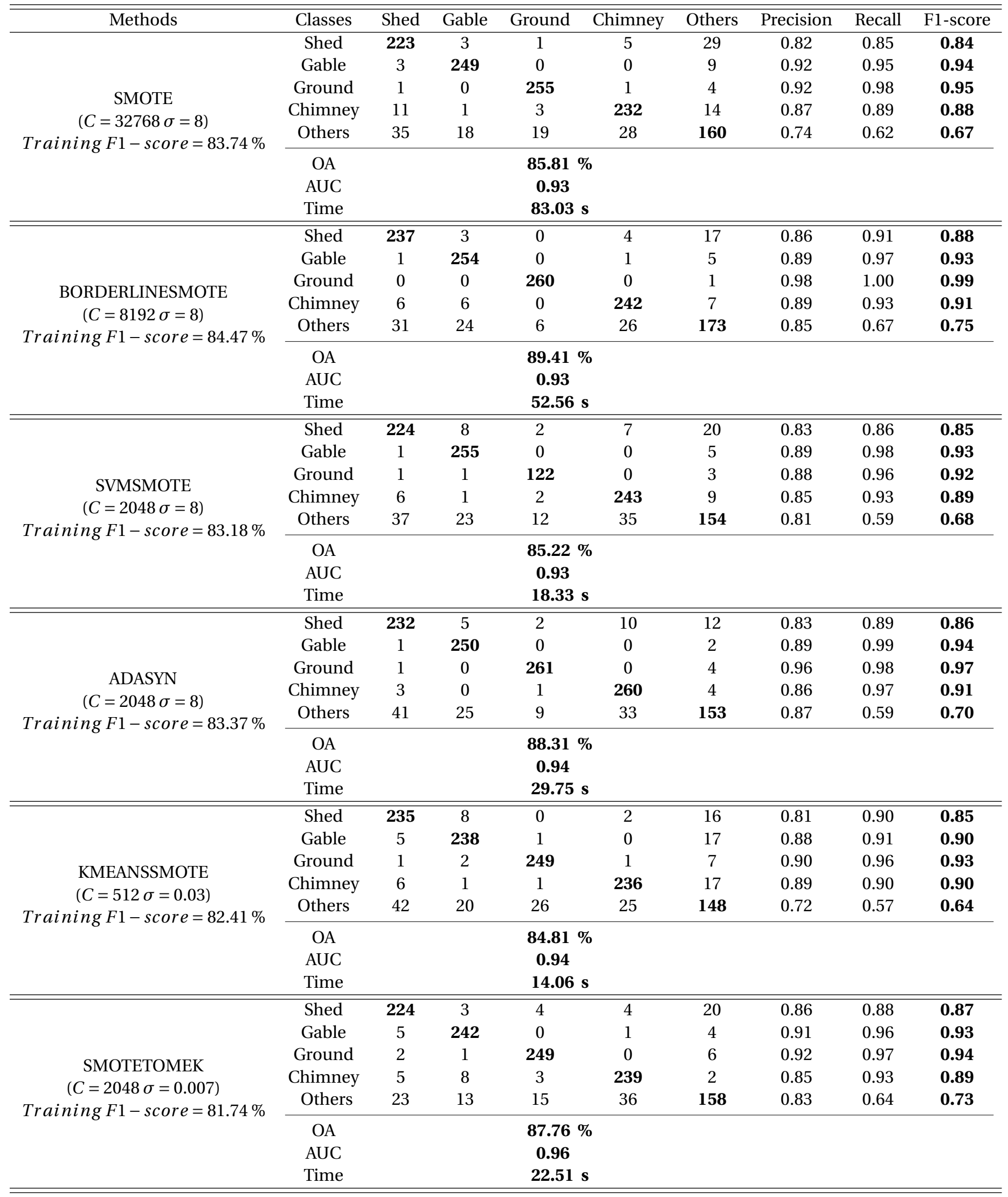


TABLE XII: PEARSON CORRELATION VALUES

\begin{tabular}{l|cccccccccccc}
\hline \hline & F1 & F2 & F3 & F4 & F5 & F6 & F7 & F8 & F9 & F10 & F11 & F12 \\
\hline F1 & $\mathbf{1 . 0 0}$ & & & & & & & & & & & \\
F2 & 0.35 & $\mathbf{1 . 0 0}$ & & & & & & & & & & \\
F3 & -0.01 & -0.10 & $\mathbf{1 . 0 0}$ & & & & & & & & & \\
F4 & 0.81 & 0.36 & -0.02 & $\mathbf{1 . 0 0}$ & & & & & & & & \\
F5 & 0.56 & 0.11 & -0.03 & 0.64 & $\mathbf{1 . 0 0}$ & & & & & & & \\
F6 & -0.01 & -0.12 & -0.04 & 0.02 & 0.01 & $\mathbf{1 . 0 0}$ & & & & & & \\
F7 & 0.01 & -0.08 & -0.05 & 0.04 & 0.02 & $\mathbf{1 . 0 0}$ & $\mathbf{1 . 0 0}$ & & & & & \\
F8 & 0.00 & -0.10 & -0.05 & 0.03 & 0.01 & $\mathbf{1 . 0 0}$ & $\mathbf{1 . 0 0}$ & $\mathbf{1 . 0 0}$ & & & & \\
F9 & -0.33 & -0.32 & 0.04 & -0.28 & -0.09 & 0.07 & 0.06 & 0.06 & $\mathbf{1 . 0 0}$ & & & \\
F10 & 0.77 & 0.44 & 0.03 & 0.61 & 0.23 & -0.02 & -0.00 & -0.01 & -0.48 & $\mathbf{1 . 0 0}$ & & \\
F11 & 0.22 & 0.95 & -0.10 & 0.25 & 0.06 & -0.11 & -0.07 & -0.09 & -0.25 & 0.27 & $\mathbf{1 . 0 0}$ & \\
F12 & 0.21 & $\mathbf{0 . 9 4}$ & -0.10 & 0.24 & 0.05 & -0.21 & -0.17 & -0.19 & -0.25 & 0.26 & $\mathbf{0 . 9 9}$ & $\mathbf{1 . 0 0}$ \\
\hline \hline
\end{tabular}

TABLE XIII: COMPARISON OF DIFFERENT CLASSIFIERS

\begin{tabular}{|l|ccc|ccc|ccc|ccc|}
\hline \hline Methods & \multicolumn{4}{|c|}{ SVM } & \multicolumn{3}{c|}{ Random Forest } & \multicolumn{3}{c|}{ Decision Tree } & \multicolumn{3}{c|}{ AdaBoost } \\
\hline Metrics & OA(\%) & AUC & Time (s) & OA(\%) & AUC & Time (s) & OA(\%) & AUC & Time (s) & OA(\%) & AUC & Time (s) \\
\hline CSBS & $\mathbf{8 8 . 2 8}$ & $\mathbf{0 . 9 6}$ & $\mathbf{9 . 9}$ & $\mathbf{8 5 . 9 3}$ & $\mathbf{0 . 9 6}$ & $\mathbf{2 . 1 7}$ & $\mathbf{8 7 . 2 1}$ & $\mathbf{0 . 9 5}$ & $\mathbf{0 . 0 2}$ & 0.08 & 0.45 & 3.99 \\
SMOTE & 87.11 & 0.96 & 8.15 & 78.22 & 0.86 & 5.64 & 72.69 & 0.82 & 0.1 & 18.48 & 0.47 & 0.35 \\
SVMSMOTE & 85.90 & 0.96 & 8.43 & 77.79 & 0.87 & 6.67 & 77.11 & 0.86 & 0.09 & 26.04 & 0.49 & 0.2 \\
ADASYN & 83.90 & 0.92 & 334.73 & 77.54 & 0.85 & 2.44 & 72.41 & 0.82 & 0.69 & 15.24 & 0.45 & 0.23 \\
BORDER-SMOTE & 85.66 & 0.92 & 262.44 & 80.59 & 0.87 & 0.79 & 74.92 & 0.84 & 0.09 & 14.72 & 0.44 & 0.18 \\
KMEANSSMOTE & 84.81 & 0.94 & 11.64 & 75.84 & 0.85 & 0.80 & 71.54 & 0.82 & 0.09 & 17.94 & 0.46 & 0.29 \\
SMOTETOMEK & 86.94 & 0.95 & 10.64 & 79.35 & 0.86 & 2.29 & 75.07 & 0.84 & 0.09 & 17.16 & 0.46 & 0.29 \\
\hline \hline
\end{tabular}

clouds," ISPRS journal of photogrammetry and remote sensing, vol. 144, pp. 400-411, 2018.

[6] S.-I. Oh and H.-B. Kang, "Fast occupancy grid filtering using grid cell clusters from lidar and stereo vision sensor data," IEEE Sensors Journal, vol. 16, no. 19, pp. 7258-7266, 2016.

[7] C. Ippolito, K. Krishnakumar, and S. Hening, "Preliminary results of powerline reconstruction from airborne lidar for safe autonomous low-altitude urban operations of small uas," in 2016 IEEE SENSORS. IEEE, 2016, pp. 1-3.

[8] T. Sun, M. Liu, H. Ye, and D.-Y. Yeung, "Point-cloud-based place recognition using cnn feature extraction," IEEE Sensors Journal, vol. 19, no. 24, pp. 12 175-12 186, 2019.

[9] X. T. Nguyen, H.-J. Lee, H. Kim et al., "A high-definition lidar system based on two-mirror deflection scanners," IEEE Sensors Journal, vol. 18, no. 2, pp. 559-568, 2017.

[10] G. Zhou, X. Zhou, J. Yang, Y. Tao, X. Nong, and O. Baysal, "Flash lidar sensor using fiber-coupled apds," IEEE Sensors Journal, vol. 15, no. 9, pp. 4758-4768, 2015.

[11] S. Zorzi, E. Maset, A. Fusiello, and F. Crosilla, "Full-waveform airborne lidar data classification using convolutional neural networks," IEEE transactions on geoscience and remote sensing, vol. 57, no. 10, pp. 8255-8261, 2019.

[12] P. H. Akwensi, Z. Kang, and J. Yang, "Fisher vector encoding of supervoxel-based features for airborne lidar data classification," IEEE Geoscience and Remote Sensing Letters, 2019.

[13] M. Awrangjeb and C. S. Fraser, "Automatic segmentation of raw lidar data for extraction of building roofs," Remote Sensing, vol. 6, no. 5, pp. 3716-3751, 2014.

[14] W. Y. Yan, A. Shaker, and N. El-Ashmawy, "Urban land cover classification using airborne lidar data: A review," Remote Sensing of Environment, vol. 158, pp. 295-310, 2015.

[15] Y. Gu, Q. Wang, and B. Xie, "Multiple kernel sparse representation for airborne lidar data classification," IEEE Transactions on Geoscience and Remote Sensing, vol. 55, no. 2, pp. 1085-1105, 2016.

[16] S. O. Elberink and G. Vosselman, "Entities and features for classifcation of airborne laser scanning data in urban area," ISPRS Annals Photogramm Remote Sens Spat Information Sciences, pp. 257-262, 2012.
[17] G. Vosselman, M. Coenen, and F. Rottensteiner, "Contextual segmentbased classification of airborne laser scanner data," ISPRS journal of photogrammetry and remote sensing, vol. 128, pp. 354-371, 2017.

[18] G. Vosselman, B. G. Gorte, G. Sithole, and T. Rabbani, "Recognising structure in laser scanner point clouds," International archives of photogrammetry, remote sensing and spatial information sciences, vol. 46, no. 8, pp. 33-38, 2004.

[19] T. Rabbani, "Automatic reconstruction of industrial installations using point clouds and images," Publications on Geodesy, 62, 2006.

[20] G. Forlani, C. Nardinocchi, M. Scaioni, and P. Zingaretti, "Building reconstruction and visualization from lidar data," International Archives Of Photogrammetry Remote Sensing And Spatial Information Sciences, vol. 34, no. 5/W12, pp. 151-156, 2003.

[21] A. Habib and Y.-J. Lin, "Multi-class simultaneous adaptive segmentation and quality control of point cloud data," Remote Sensing, vol. 8, no. 2, p. 104, 2016

[22] A.-V. Vo, L. Truong-Hong, D. F. Laefer, and M. Bertolotto, "Octreebased region growing for point cloud segmentation," ISPRS Journal of Photogrammetry and Remote Sensing, vol. 104, pp. 88-100, 2015.

[23] T. Melzer, "Non-parametric segmentation of als point clouds using mean shift," Journal of Applied Geodesy Jag, vol. 1, no. 3, pp. 159170, 2007.

[24] M. ComaniciuD, "A robust approach toward feature spaceanalysis [j]," IEEE Transactions on Pattern Analysis and Machine Intelligence, vol. 24, no. 5, pp. 313-329, 2002.

[25] M. Rutzinger, B. Höfle, M. Hollaus, and N. Pfeifer, "Object-based point cloud analysis of full-waveform airborne laser scanning data for urban vegetation classification," Sensors, vol. 8, no. 8, pp. 45054528, 2008.

[26] S. A. N. Gilani, M. Awrangjeb, and G. Lu, "Segmentation of airborne point cloud data for automatic building roof extraction," GIScience \& remote sensing, vol. 55, no. 1, pp. 63-89, 2018.

[27] A. Jochem, B. Höfle, V. Wichmann, M. Rutzinger, and A. Zipf, "Areawide roof plane segmentation in airborne lidar point clouds," Computers, Environment and Urban Systems, vol. 36, no. 1, pp. 54-64, 2012.

[28] M. Kada and A. Wichmann, "Sub-surface growing and boundary generalization for 3d building reconstruction," ISPRS Annals of the 
Photogrammetry, Remote Sensing and Spatial Information Sciences I3, pp. 233-238, 2012.

[29] S. I. Zolanvari, D. F. Laefer, and A. S. Natanzi, "Three-dimensional building façade segmentation and opening area detection from point clouds," ISPRS journal of photogrammetry and remote sensing, vol. 143, pp. 134-149, 2018.

[30] H. Fan, W. Yao, and Q. Fu, "Segmentation of sloped roofs from airborne lidar point clouds using ridge-based hierarchical decomposition," Remote Sensing, vol. 6, no. 4, pp. 3284-3301, 2014.

[31] H.-G. Maas and G. Vosselman, "Two algorithms for extracting building models from raw laser altimetry data," ISPRS Journal of photogrammetry and remote sensing, vol. 54, no. 2-3, pp. 153-163, 1999.

[32] M. Brédif, D. Boldo, M. Pierrot-Deseilligny, and H. Maître, "3d building reconstruction with parametric roof superstructures," in 2007 IEEE International Conference on Image Processing, vol. 2. IEEE, 2007, pp. II-537.

[33] E. Byon, A. K. Shrivastava, and Y. Ding, "A classification procedure for highly imbalanced class sizes," IIE Transactions, vol. 42, no. 4, pp. 288-303, 2010.

[34] D.-C. Li, H.-Y. Chen, and Q.-S. Shi, "Learning from small datasets containing nominal attributes," Neurocomputing, vol. 291, pp. 226236, 2018.

[35] M. Azadbakht, C. Fraser, and K. Khoshelham, "Improved urban scene classification using full-waveform lidar," Photogrammetric Engineering \& Remote Sensing, vol. 82, no. 12, pp. 973-980, 2016.

[36] F. H. Nahhas, H. Z. Shafri, M. I. Sameen, B. Pradhan, and S. Mansor, "Deep learning approach for building detection using lidarorthophoto fusion," Journal of Sensors, vol. 2018, 2018.

[37] G. Miliaresis and N. Kokkas, "Segmentation and object-based classification for the extraction of the building class from lidar dems," Computers \& Geosciences, vol. 33, no. 8, pp. 1076-1087, 2007.

[38] D. O. Rubio, A. Lenskiy, and J.-H. Ryu, "Connected components for a fast and robust 2d lidar data segmentation," in 2013 7th Asia Modelling Symposium. IEEE, 2013, pp. 160-165.

[39] J. Zhang, X. Lin, and X. Ning, "Svm-based classification of segmented airborne lidar point clouds in urban areas," Remote Sensing, vol. 5, no. 8, pp. 3749-3775, 2013.

[40] W. Xiao, S. Xu, S. O. Elberink, and G. Vosselman, "Individual tree crown modeling and change detection from airborne lidar data," IEEE Journal of selected topics in applied earth observations and remote sensing, vol. 9, no. 8, pp. 3467-3477, 2016.

[41] G. Vosselman, "Point cloud segmentation for urban scene classification," Int. Arch. Photogramm. Remote Sens. Spat. Inf. Sci, vol. 1, pp. 257-262, 2013.

[42] H. Haibo, B. Yang, G. A. Edwardo, and L. Shutao, "Adaptive synthetic sampling approach for imbalanced learning," in IEEE International Joint Conference on Neural Networks, IJCNN, vol. 8, no. 3, 2008, pp. 1322-1328.

[43] N. V. Chawla, K. W. Bowyer, L. O. Hall, and W. P. Kegelmeyer, "Smote: synthetic minority over-sampling technique," Journal of artificial intelligence research, vol. 16, pp. 321-357, 2002.

[44] H. Han, W.-Y. Wang, and B.-H. Mao, "Borderline-smote: a new oversampling method in imbalanced data sets learning," in International conference on intelligent computing. Springer, 2005, pp. 878-887.

[45] H. M. Nguyen, E. W. Cooper, and K. Kamei, "Borderline over-sampling for imbalanced data classification," in Proceedings: Fifth International Workshop on Computational Intelligence \& Applications, vol. 2009, no. 1. IEEE SMC Hiroshima Chapter, 2009, pp. 24-29.

[46] G. Douzas, F. Bacao, and F. Last, "Improving imbalanced learning through a heuristic oversampling method based on k-means and smote," Information Sciences, vol. 465, pp. 1-20, 2018.

[47] Z. Wang, C. Wu, K. Zheng, X. Niu, and X. Wang, "Smotetomekbased resampling for personality recognition," IEEE Access, vol. 7, pp. 129678-129689, 2019.

[48] B. Aissou and A. Belhadj Aissa, "An adapted connected component labeling for clustering non-planar objects from airborne lidar point cloud," ISPRS-International Archives of the Photogrammetry, Remote Sensing and Spatial Information Sciences, vol. 43, pp. 191-195, 2020.

[49] Z. Zhang, L. Zhang, X. Tong, P. T. Mathiopoulos, B. Guo, X. Huang, Z. Wang, and Y. Wang, "A multilevel point-cluster-based discriminative feature for als point cloud classification," IEEE Transactions on Geoscience and Remote Sensing, vol. 54, no. 6, pp. 3309-3321, 2016.

[50] J. Im, J. R. Jensen, and M. E. Hodgson, "Object-based land cover classification using high-posting-density lidar data," GIScience \& Remote Sensing, vol. 45, no. 2, pp. 209-228, 2008.
[51] A. Nandy, R. Chakraborty, P. Chakraborty, and G. C. Nandi, "A novel approach to human gait recognition using possible speed invariant features," International Journal of Computational Intelligence Systems, vol. 7, no. 6, pp. 1174-1193, 2014.

[52] H. Edelsbrunner, D. Kirkpatrick, and R. Seidel, "On the shape of a set of points in the plane," IEEE Transactions on information theory, vol. 29, no. 4, pp. 551-559, 1983.

[53] B. Guo, X. Huang, F. Zhang, and G. Sohn, "Classification of airborne laser scanning data using jointboost," ISPRS Journal of Photogrammetry and Remote Sensing, vol. 100, pp. 71-83, 2015.

[54] Z. I. Petrou, I. Manakos, T. Stathaki, C. A. Mücher, and M. Adamo, "Discrimination of vegetation height categories with passive satellite sensor imagery using texture analysis," IEEE Journal of Selected Topics in Applied Earth Observations and Remote Sensing, vol. 8, no. 4, pp. 1442-1455, 2015.

[55] M. Cramer, "The dgpf-test on digital airborne camera evaluationoverview and test design," Photogrammetrie-FernerkundungGeoinformation, vol. 2010, no. 2, pp. 73-82, 2010.

[56] T. Kavzoglu and I. Colkesen, "A kernel functions analysis for support vector machines for land cover classification," International Journal of Applied Earth Observation and Geoinformation, vol. 11, no. 5, pp. 352-359, 2009.

[57] M. M. Awad, "Toward robust segmentation results based on fusion methods for very high resolution optical image and lidar data," IEEE Journal of Selected Topics in Applied Earth Observations and Remote Sensing, vol. 10, no. 5, pp. 2067-2076, 2017.

[58] C. Mallet, F. Bretar, M. Roux, U. Soergel, and C. Heipke, "Relevance assessment of full-waveform lidar data for urban area classification," ISPRS journal of photogrammetry and remote sensing, vol. 66, no. 6 , pp. S71-S84, 2011.

[59] N. Yokoya, P. Ghamisi, J. Xia, S. Sukhanov, R. Heremans, I. Tankoyeu, B. Bechtel, B. Le Saux, G. Moser, and D. Tuia, "Open data for global multimodal land use classification: Outcome of the 2017 ieee grss data fusion contest," IEEE Journal of Selected Topics in Applied Earth Observations and Remote Sensing, vol. 11, no. 5, pp. 1363-1377, 2018.

[60] Z. Kang and J. Yang, "A probabilistic graphical model for the classification of mobile lidar point clouds," ISPRS journal of photogrammetry and remote sensing, vol. 143, pp. 108-123, 2018.

[61] J. Niemeyer, F. Rottensteiner, and U. Soergel, "Contextual classification of lidar data and building object detection in urban areas," ISPRS journal of photogrammetry and remote sensing, vol. 87, pp. 152-165, 2014.

[62] Z. Li, L. Zhang, R. Zhong, T. Fang, L. Zhang, and Z. Zhang, "Classification of urban point clouds: A robust supervised approach with automatically generating training data," IEEE Journal of Selected Topics in Applied Earth Observations and Remote Sensing, vol. 10, no. 3, pp. 1207-1220, 2016.

[63] N. Li, C. Liu, and N. Pfeifer, "Improving lidar classification accuracy by contextual label smoothing in post-processing," ISPRS journal of photogrammetry and remote sensing, vol. 148, pp. 13-31, 2019.

[64] N. Crasto, C. Hopkinson, D. Forbes, L. Lesack, P. Marsh, I. Spooner, and J. Van Der Sanden, "A lidar-based decision-tree classification of open water surfaces in an arctic delta," Remote Sensing of Environment, vol. 164, pp. 90-102, 2015.

[65] Z. Li, L. Zhang, X. Tong, B. Du, Y. Wang, L. Zhang, Z. Zhang, H. Liu, J. Mei, X. Xing et al., "A three-step approach for tls point cloud classification," IEEE Transactions on Geoscience and Remote Sensing, vol. 54, no. 9, pp. 5412-5424, 2016.

[66] Y. Wang, Q. Chen, L. Liu, X. Li, A. K. Sangaiah, and K. Li, "Systematic comparison of power line classification methods from als and mls point cloud data," Remote Sensing, vol. 10, no. 8, p. 1222, 2018.

[67] L. Landrieu, H. Raguet, B. Vallet, C. Mallet, and M. Weinmann, "A structured regularization framework for spatially smoothing semantic labelings of 3d point clouds," ISPRS Journal of Photogrammetry and Remote Sensing, vol. 132, pp. 102-118, 2017.

[68] S. Xu, G. Vosselman, and S. O. Elberink, "Multiple-entity based classification of airborne laser scanning data in urban areas," ISPRS Journal of photogrammetry and remote sensing, vol. 88, pp. 1-15, 2014.

[69] Y. Li, G. Tong, X. Du, X. Yang, J. Zhang, and L. Yang, "A single point-based multilevel features fusion and pyramid neighborhood optimization method for als point cloud classification," Applied Sciences, vol. 9, no. 5, p. 951, 2019.

[70] D. Su, F. Yang, Y. Ma, K. Zhang, J. Huang, and M. Wang, "Classification of coral reefs in the south china sea by combining airborne lidar bathymetry bottom waveforms and bathymetric features," IEEE Transactions on Geoscience and Remote Sensing, vol. 57, no. 2, pp. 815-828, 2018. 